\title{
Use of antivascular endothelial growth factor for diabetic macular edema
}

This article was published in the following Dove Press journal:

Clinical Ophthalmology

22 May 2010

Number of times this article has been viewed

\author{
Rushmia Karim \\ Benjamin Tang \\ University of Sydney School of Public \\ Health, Concord Repatriation General \\ Hospital, Concord, NSW, Australia
}

Background: Diabetic macular edema (DME) is one of the manifestations of diabetic retinopathy leading to loss of central vision and visual acuity. It manifests itself with swelling around the central part of the retina, the area responsible for sharp vision. Current treatment includes laser therapy and intravitreal steroids with preventative measures including diabetes control. No one treatment has guaranteed control of diabetic macular edema which leads to deteriorating visual acuity, function and quality of life in patients. Vascular endothelial growth factor (VEGF) has been shown to be a critical stimulus in the pathogenesis of macular edema secondary to diabetes. ${ }^{1}$ Antiangiogenic therapy encompassed treatment with anti-VEGF which inhibits VEGF-driven neovascularization hence macular edema leading to decreased visual acuity.

Objective: For this review, we evaluated the effectiveness of intravitreal anti-VEGF in treating DME.

Data sources: We identified five trials $(\mathrm{n}=525)$ using electronic databases (Cochrane Central Register of Controlled Trials [Central], Medline ${ }^{\circledR}$, and Excerpta Medica Database [EMBASE ${ }^{\circledR}$ ]) in October 2008, supplemented by hand searching of reference lists, review articles, and conference abstracts.

Methods: We included all randomized clinical trials (RCTs) evaluating any form of intravitreal anti-VEGF for treating DME. The main outcome factor was change in best-corrected visual acuity and central macular thickness. One author assessed eligibility, methodological quality, and extracted data. Meta analysis was performed when appropriate.

Results: We included three trials of adequate methodological quality in our metaanalysis. Patients treated with anti-VEGF showed improvement in visual acuity of -0.17 (95\% confidence interval [CI]: $-0.23,-0.10)$ and central macular thickness -84.69 (95\% CI: $-117.09,-52.30)$. Patients treated with combined anti-VEGF and intravitreal triamcinolone showed improvement of visual acuity of -0.19 (95\% CI: $-0.27,-0.11)$ and central macular thickness mean change being -111.20 (95\% CI: -148.13, -74.28).

Conclusions: Anti-VEGF has been associated with an improvement in visual acuity and central macular thickness in the analysis, however trial analysis was of a short duration and further research is needed to determine long-term benefits.

Keywords: anti-VEGF, diabetic macular edema, ranizubimab, Avastin ${ }^{\circledR}$, pegaptanib

\section{Introduction}

Diabetic macular edema (DME) is one of the manifestations of diabetic retinopathy leading to loss of central vision and visual acuity. ${ }^{2}$ DME results from microvascular changes in the retina in both type 1 and type 2 diabetes mellitus. ${ }^{3}$ DME is defined as thickening located within two disc diameters of the center of the macula. When this is 
present within or close to the central macula, it is termed clinically significant macular edema (CSME). ${ }^{4} \mathrm{DME}$ is further classified descriptively into focal and diffuse DME. Focal DME describes the edema from individual microaneurysms where as diffuse DME implies extensive or generalized leakage from the posterior capillary bed and breakdown of the inner blood-retinal barrier. Combinations of the two are frequent.

It is important to detect DME in the assessment of diabetic retinopathy as this is the most frequent cause of decreased vision from retinopathy. Both macular edema (ME) and CSME, defined by proximity of these signs to the foveal center, are best assessed using slit-lamp biomicroscopy or by grading stereoscopic macular photographs. Optical coherence tomography (OCT) may be also used to provide valuable confirmation and quantification of the clinical grading for DME and facilitate monitoring of its response to therapy. ${ }^{4}$

This review is concerned with diabetic macular edema, both focal and diffuse. The treatment considered in this review is anti-vascular endothelial growth factor (antiVEGF).

\section{Epidemiology}

Between 25\% and $44 \%$ of people with diabetes have some form of diabetic retinopathy (DR) at any point in time. The United States National Eye Institute pooled data from eight well-conducted population-based studies of persons aged 40 years or older with consistent retinopathy grading from retinal photographs. Data included that from five United States (US) studies, one West Indian study, and two Australian studies (Blue Mountains Eye Study [BMES] and Melbourne Visual Impairment Project [MVIP]). The overall crude DR prevalence was $40 \%$. The prevalence of sight-threatening retinopathy (CSME or proliferative diabetic retinopathy [PDR]) was $8.2 \%$. The general US population prevalence of DR and sight-threatening retinopathy were 3.4\% (4.1 million persons) and $0.8 \%$ (900,000 persons), respectively. Projected to the current Australian population, these rates suggest a prevalence of 300,000 and 65,000, respectively, for any DR and sight-threatening retinopathy (PDR or CSME) in persons aged over 40 years. $^{4}$

\section{Clinical presentation and diagnosis}

Blurred vision is the most common clinical presentation of DME, as well as distortion of the visual image (metamorphopsia), floaters, and changes in contrast sensitivity. Photophobia, changes in color vision, and scotoma occurs in DME while a loss of vision is associated with increased severity and progression of the disease. Chronic macular edema can be associated with cystoid macular edema.

Stereoscopic observation of the macular is considered the most acceptable way to diagnose DME, however use of OCT, stereofundus photography and fluorescein angiography have become acceptable tools for diagnosis in clinical practice. ${ }^{5}$

\section{Treatment options}

Strict glycemic control is the hallmark of prevention and halts progression of disease. Laser photocoagulation is used to coagulate tissue either by direct focal photocoagulation for focal macular edema or using grid photocoagulation for diffuse DME. The Early Treatment Diabetic Retinopathy Study (ETDRS) demonstrated that photocoagulation reduced the risk of moderate vision loss, especially for those eyes with macular edema that involved or threatened the center of the macular. ${ }^{6}$ This has become a standard treatment in clinical practice. Steroids including intravitreal triamcinolone (IVT) or surgical implantation have increased in popularity for treatment of DME due to their angiostatic effects and antipermeabilty properties which minimize systemic toxicity. Vitrectomy is considered for treatment in eyes with chronic or diffuse DME that is not responsive to laser. ${ }^{7}$

\section{Pathophysiology and intervention}

Vascular endothelial growth factor (VEGF); also known as VEGF-A) has an important role in angiogenesis (ie, the migration and mitosis of endothelial cells) up-regulating methane monooxygenase and $\alpha v \beta 3$ activity, and the creation of blood vessel lumen and fenestrations. As seen in preclinical models, VEGF has been shown to facilitate survival of existing vessels, contribute to vascular abnormalities (eg, tortuousness and hyper permeability) that may impede effective delivery of antitumor compounds, and stimulate new vessel growth. ${ }^{8}$

VEGF has been shown to be an endothelial cell specific mitogen, an angiogenic inducer, and is also known to increase retinal vessel permeability. ${ }^{2}$ Hypoxia-regulated VEGF release likely plays a key role in the normal development of the retina and, given its hypoxia inducibility, VEGF became an attractive candidate as a mediator of pathological intraocular neovascularization. Expression of VEGF messenger ribonucleic acid (mRNA) spatially and temporally correlates with neovascularization in several animal models of retinal ischemia. ${ }^{33}$ Elevations of VEGF levels in the aqueous and vitreous humor of human eyes with proliferative retinopathy secondary to diabetes, and other conditions have been previously described. ${ }^{9}$ 
Anti-VEGF modalities have been shown to potently suppress angiogenesis and growth in a variety of human tumor cells lines and also to inhibit neovascularization of ischemic retinal disease.

Bevacizumab is a full-length humanized monoclonal antibody against $\mathrm{VEGF}^{10}$ meaning it binds to all subtypes of VEGF. Pegaptanib is a synthesized anti-VEGF aptamer of a single ribonucleic acid strand that specifically targets VEGF-165 and binds only to it. Aptamers are oligonucleotide ligands that are selected for high-affinity binding to molecular targets. ${ }^{11}$ Ranibizumab (rhuFab-VEGF) is an antibody fragment which neutralizes all VEGF isoforms and bioactive fragments.

Recent studies have demonstrated the usefulness of anti-VEGF in the reduction of macular edema secondary to central retinal vein occlusion, vascular permeability, and fibrovascular proliferation in retinal neovascularization secondary to PDR, and choroidal neovascularization secondary to aged-related macular degeneration (AMD). ${ }^{12}$

\section{Rationale for a systematic review}

Monoclonal antibodies against vascular endothelial growth factor (VEGF) were first developed in treatment of metastatic colorectal cancer. ${ }^{13}$ Anti-vascular endothelial growth factor is now commonly used for age-related macular degeneration to halt progression of abnormal growth of blood vessels in the back of the eye. A Cochrane review of five RCTs concluded the use of two anti-VEGFs to reduce the risk of visual acuity loss in neovascular age-related macular degeneration. ${ }^{14}$ More recently, these results have prompted trials in applying anti-VEGF in diabetic macular edema and macular edema secondary to central retinal vein occlusion.

In a 6-month follow-up study for anatomic and bestcorrected visual (BCVA) acuity after primary intravitreal bevacizumab $\left(\right.$ Avastin $^{\circledR}$ ) in patients with DME, Arevalo et $\mathrm{al}^{2}$ showed improvements in visual acuity, OCT, and fluorescan angiography (FA) for all doses injected. Nguyen et al ${ }^{1}$ showed promising results with ranibizumab (a specific antagonist of VEGF) in a nonrandomized clinical trial. Results showed intraocular injections of ranibizumab significantly reduced foveal thickness and improved visual acuity in 10 patients with DME.

In a retrospective review Chung et $\mathrm{a}^{15}$ concluded a decrease in mean visual acuity score after three months in an ischemic subgroup (patients with an enlarged foveal avascular zone [FAZ] or broken perifoveal capillary ring at the border of the FAZ, with a distinct area of capillary nonperfusion).
Their findings indicate the use of anti-VEGF has a negative effect on short-term visual outcome in a particular subgroup of DME.

DME continues to progress in spite of a lack of a gold standard treatment, although options of vitrectomy, laser photocoagulation, and the emerging popularity of intravitreal steroids have been shown to halt progression of disease.

Although the ETDRS demonstrated that immediate focal photocoagulation reduced moderate visual loss by $50 \%$ (from $24 \%$ to $12 \%, 3$ years after initiation of treatment), $12 \%$ of treated eyes still lost $>15$ ETDRS letters at 3 year follow-up. Approximately $40 \%$ of treated eyes with retinal thickening involving the center of the macula at baseline still had thickening involving the center at 12 months. Only $3 \%$ of laser-treated eyes experienced a gain of $>3$ lines of vision. ${ }^{6}$

Anti-VEGF provides an option to treatment for these patients and it may also be a very useful adjunctive treatment before laser or vitrectomy surgery or a potentially important role as an adjunct to laser in the management of DME. Recently, RCTs have been published and continued to examine various antiangiogenic therapies. There has been no systematic review published evaluating RCTs conducted with treatment of VEGF for DME. Given the disease burden and significance of vision in terms of quality of life, a systematic review is needed to examine the evidence regarding the effectiveness and safety of antiangiogenic therapy with anti-VEGF modalities for treatment of DME.

\section{Objective}

The objective of this review was to assess the effectiveness of antiangiogenic therapy with anti-VEGF modalities in the treatment of diabetic macular edema. Tables 1 and 2 show inclusion and exclusion criteria for the studies considered for this review.

\section{Data sources}

We searched the Cochrane Central Register of Controlled Trials (Central) (which contains the Cochrane Eyes and Vision Group Trials Register) in the Cochrane Library, Medline $^{\circledR}$ and EMBASE $^{\circledR}$. There were no language or data restrictions in the search for trials. The databases were last searched on October 9, 2008. Reference lists of included trials were searched. The Australian National Health and Medical Research Council (NHMRC) guidelines for diabetic retinopathy references were searched. Archives of Ophthalmology, Ophthalmology, Retina, and the New England Journal of Medicine were searched for clinical trials and 
Table I Inclusion criteria for considering studies for this review

Types of studies

Participants

Interventions

Outcome measures

Adverse effects

Quality of life measures

Economic data

Abbreviations: BCVA, best-corrected visual acuity; DME, diabetic macular edema; anti-VEGF, anti-vascular endothelial growth factor; ETDRS, Early Treatment Diabetic Retinopathy Study.

reviews. Hand-searching of references and their associated clinical trials was conducted.

We also searched for unpublished clinical trials and those in progress using clinical trials repositories including the National Institute of Health repository, ${ }^{16}$ the Current Controlled Trials repository, ${ }^{17}$ and the National Research

Table 2 Exclusion criteria for considering studies for this review Exclusion criteria

RCTs for interventions of VEGF for diabetic
retinopathy with no mention of diabetic macular
edema or clinically significant diabetic macular
edema were excluded in the analysis.
Studies of macular edema due to another cause
other than DME were excluded.
Full text was reviewed and discussed.
Studies that were not RCT.

Abbreviations: $\mathrm{RCT}$, randomized clinical trials; VEGF, vascular endothelial growth factor; DME, diabetic macular edema.
Register Repository. ${ }^{18}$ Authors were contacted of unpublished closed trials for initial results. For full search details, see Appendix.

\section{Selection of studies}

Screening of titles and abstracts resulting from electronic and manual searches were reviewed. Abstracts were classified as relevant, potentially relevant, or not relevant for this review. Full copies of abstracts were obtained for relevant and potentially relevant reviews. Abstracts and full reviews were read to determine inclusion. Only randomized clinical trials were eligible. Study findings are in concordance with the Quorom statement. Figure 1 illustrates this selection of studies with a flow diagram.

\section{Methods}

\section{Data extraction and management}

Table 3 illustrates extracted data for the primary and secondary outcomes for this review.

\section{Data synthesis}

BCVA and central macular thickness (CMT), the primary outcome variables, are expressed as continuous variables. Standard deviations were calculated by Cunningham et al using actual $P$ values obtained from $t$-tests quoted by Cochrane. $^{19}$

For every study, we calculated the mean difference for the primary outcome BCVA, LogMAR, and the CMT using 95\% confidence intervals. The outcome measures were pooled by use of the fixed-effect model as there were only three trials used in the meta-analysis.

Heterogeneity was calculated using Cochran's Q statistic and quantified using the $\mathrm{I}_{2}$ statistic. These indicated the proportion of variability across studies due to heterogeneity, rather than sample error. Despite a high $\mathrm{I}_{2}$, results were pooled as examination of these studies on a forest plot indicated that the individual trial results were consistent in the direction of the effect (ie, the mean difference and confidence intervals largely fell on one side of the null line).

Clinical heterogeneity was present between the studies in relation to dosage and type of anti-VEGF use. Treatment duration and follow-up varied from 12 to 36 weeks. Despite clinical heterogeneity, trials were pooled and overall efficacy from any type of dose or duration of anti-VEGF was assessed in the objectives. Subgroup analysis was not performed due to the limited trials. Characteristics of age, gender ratios, and baseline visual acuity were similar across all trials, 


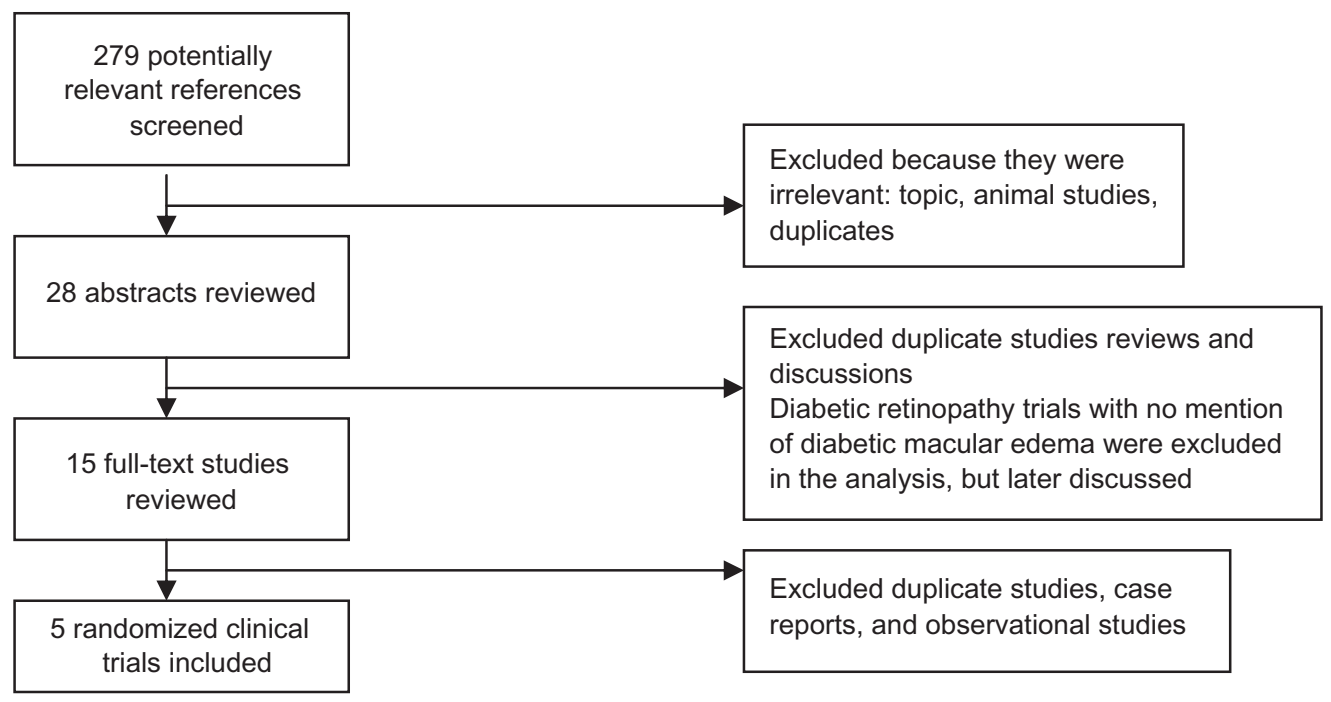

Figure I Selection of studies flow diagram.

however variability in trial quality and intervention type, dose, and timing of administration varied. Table 4 highlights the characteristics of the included studies.

Asymmetry assessment of the funnel plot was not conducted for publication bias as only three trials were used in the final analysis. In future analyses, asymmetry of the funnel plot will be used to identify publication bias if at least seven studies are used. In the case of missing data, efforts to contact authors were made. Data was entered in Review Manager 5 (Cochrane, Sanfrancisco, CA) and fixed effect models were used.

Assessment of risk of bias in included studies has been considered using methods described in chapter 6 of the Cochrane Handbook for Systematic Reviews of Interventions. ${ }^{19}$ The following parameters were assessed: randomization process, allocation concealment, and masking of participants and investigators. Table 4 offers a summary of included studies characteristics for trial quality assessment score.

\section{Description of studies}

Electronic searches conducted in July 2008 and October 2008 resulted in 279 abstracts with 28 abstracts reviewed. Of those, 15 full-texts were read and five were found to meet our eligibility criteria. The reference list of each of the 15 full-text articles was searched for other relevant articles. Nine studies were excluded. Table 4 provides a summary of included studies characteristics and the Appendix lists included and excluded studies. Eight publications were not RCTs and one did not mention DME with an intervention of vitrectomy surgery in the assessment and primary outcome measure.

To our knowledge, there are 10 ongoing clinical trials using anti-VEGF for treatment of DME. Authors and trial groups were contacted, but we were unable to obtain preliminary data. Cunningham et $\mathrm{al}^{21}$ was funded by a pharmaceutical company (Pfizer).

\section{Participants}

We included five studies from three countries (United States, Iran, and Brazil) with a total of 525 eyes represented in the review. The range of eye enrollments in the trials varied with the largest study enrolling 172 eyes,${ }^{21}$ however this was a study with three intervention groups and one control group. The numbers of subjects within each group, therefore, become smaller $(n=42-44)$. The study by Paccola et $\mathrm{al}^{24}$ was the smallest trial, enrolling 28 eyes.

Participants were male and female adults. All studies excluded patients who had undergone previous laser treatment at least 3-6 months prior. Soheilian et $\mathrm{a}^{23}$ included patients without prior laser treatment, whereas Ahmadieh et $\mathrm{al}^{20}$ and Paccola et $\mathrm{al}^{24}$ included participants unresponsive to previous macula laser photocoagulation at least 3 months prior. The studies by both Scott et $\mathrm{al}^{22}$ and Cunningham et $\mathrm{al}^{21}$ included patients who had had no laser treatment within 3 and 6 months, respectively.

No study included patients with other ocular conditions affecting assessment and progression of Visual acuity, such as central retinal vein occlusion, uveitis, or recent cataract surgery. All trials included patients with clinically significant 
Table 3 Extracted data

\begin{tabular}{|c|c|}
\hline \multirow[t]{11}{*}{ Participant characteristics } & Total number \\
\hline & Gender \\
\hline & Age \\
\hline & Country \\
\hline & Type of diabetic macular edema \\
\hline & Diagnostic criteria \\
\hline & Baseline visual acuity or changed in BCVA \\
\hline & Visual fields \\
\hline & Fluorescein angiography \\
\hline & $\begin{array}{l}\text { OCT-determined thickness of diabetic } \\
\text { macular edema }\end{array}$ \\
\hline & Patient inclusion and exclusion criteria \\
\hline \multirow[t]{5}{*}{ Intervention } & Agent \\
\hline & Dose \\
\hline & Timing of first dose in relation to diagnosis \\
\hline & Delivery route \\
\hline & Frequency and treatment length \\
\hline \multirow[t]{6}{*}{ Study and methodology } & Study design \\
\hline & Trial identifiers \\
\hline & Study size \\
\hline & Randomization \\
\hline & Masking, allocation concealment \\
\hline & Duration of each study \\
\hline \multirow[t]{3}{*}{ Primary outcomes } & BCVA \\
\hline & Change in visual acuity \\
\hline & OCT \\
\hline \multirow[t]{11}{*}{ Secondary outcomes } & $\begin{array}{l}\text { Retinal thickness from baseline as } \\
\text { measured by } \mathrm{OCT}^{5}\end{array}$ \\
\hline & Anatomical measures: \\
\hline & Presence of edema via direct fundoscopy \\
\hline & Fluorescein angiography leakage \\
\hline & Adverse effects: \\
\hline & Ocular and systemic toxicity \\
\hline & Ocular hypertension \\
\hline & Anterior chamber reaction \\
\hline & Lens opacity progression \\
\hline & Endophthalmitis \\
\hline & Blindness \\
\hline \multirow[t]{2}{*}{ Additional data } & Economic data, quality of life data \\
\hline & $\begin{array}{l}\text { Treatment compliance and losses to } \\
\text { follow-up }\end{array}$ \\
\hline \multirow[t]{4}{*}{ Missing data } & Authors contacted \\
\hline & Data has been entered in Review \\
\hline & Manager 5 \\
\hline & Fixed effect models used \\
\hline Data collection & Microsoft Excel ${ }^{\circledR}$ spreadsheet \\
\hline
\end{tabular}

Abbreviations: BCVA, best-corrected visual acuity; OCT, optical coherence tomography. macular edema and generally defined the edema as focal or diffuse and as persistent or refractory. All trials mentioned diabetic macular edema. None of the trials mention whether the patients also had cystic macular edema.

All of the trials explicitly report the primary outcome factor of BCVA according to the ETDRS. CMT is reported in all the studies either at baseline to follow-up measurements or as a mean change in thickness, measured in $\mu \mathrm{m}$. Duration of diabetes and baseline intraocular pressure (IOP) measurements were not explicitly mentioned in all the trials. Adverse events were described within trials. Scott et $\mathrm{al}^{22}$ presented safety summary data on adverse event rates between groups, while Ahmadieh et $\mathrm{al}^{20}$ and Soheilian et $\mathrm{al}^{23}$ described percentages of adverse reaction rates amongst the different groups. Cunningham et $\mathrm{al}^{21}$ listed adverse events among their subjects with pooled results for certain subgroups (eg, hypertension). Two-way analysis of variance was performed by Paccola et $\mathrm{al}^{24}$ for raised IOP and description of adverse effects. Adverse events described were tabled see table of adverse events but data for analysis was not performed.

\section{Interventions}

Ahmadieh et $\mathrm{al}^{20}$ was a three arm trial comparing intravitreal becavizumab (IVB) to IVB intravitreal triamcinolone acetonide (IVT) to sham. Cunningham et $\mathrm{al}^{21}$ compared three doses of IVB to sham. The Scott et $a^{22}$ study was a 5 treatment-arm trial divided as follows: a) focal laser at baseline with no intervention; b) $1.25 \mathrm{mg}$ of IVB; c) $2.5 \mathrm{mg}$ of IVB; d) baseline IVB and sham; e) and $1.25 \mathrm{mg}$ of IVB plus laser therapy. The Soheilian et $\mathrm{al}^{23}$ study was a three-arm trial comparing IVB and IVB/IVT to macular photocoagulation. Paccola et $\mathrm{al}^{24}$ compared IVB to IVT interventions.

Bevacizumab was employed in four studies and one study used pegaptanib. No studies were included using ranibizumab. Table 4 gives a summary of included studies characteristics.

\section{Outcome measures}

All trials considered visual acuity using ETDRS charts and CMT using OCT as their major outcome. Definitions of visual acuity varied across the trials. BCVA was quantified in all trials. All trials used OCT to measure CMT in $\mu \mathrm{m}$, however some studies reported CMT change whilst others reported mean baseline and follow-up results. CMT was quantified in all trials.

Adverse events of interest included: IOP increase reported in five trials; anterior chamber reaction reported in three trials; nil progression of leno pacification reported in all trials; iris neovascularization reported in three trials; 


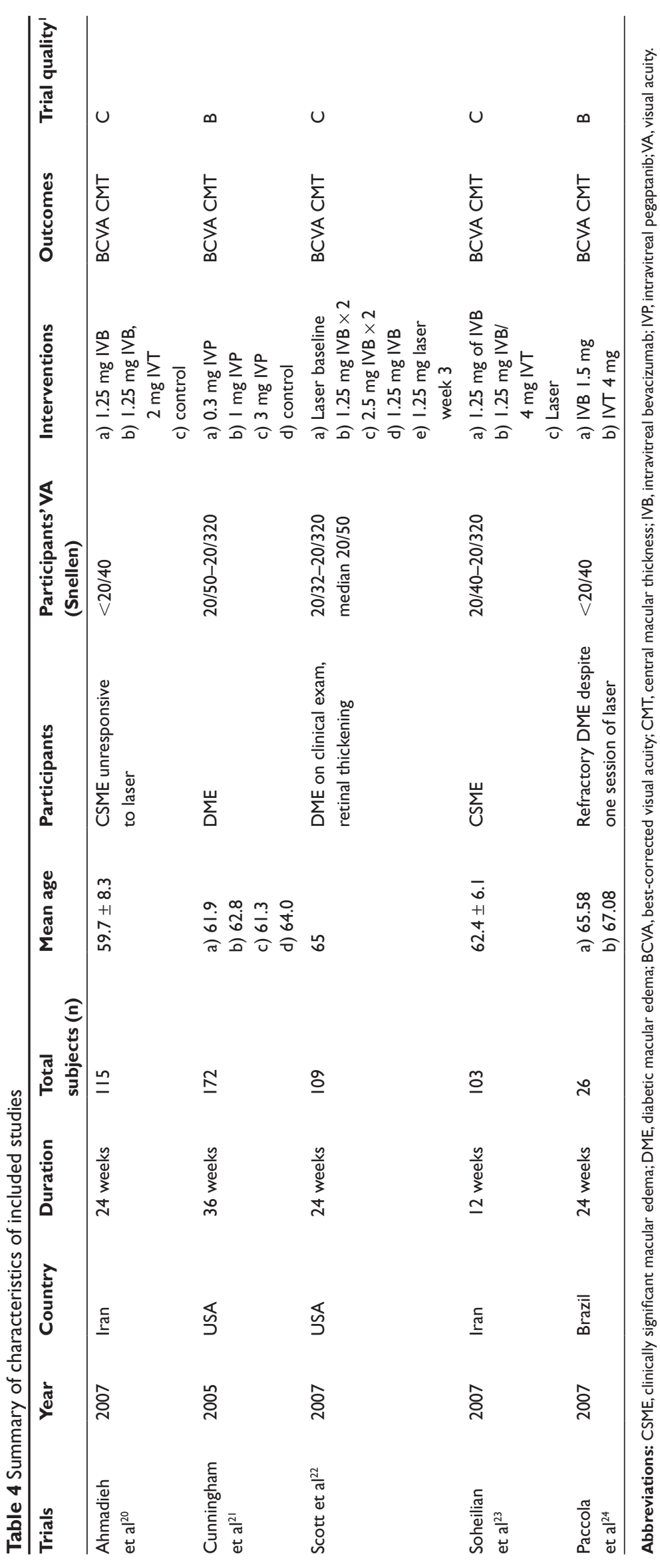


and endophthalmitis, which was considered in all trialsm but only reported as events in two trials. There was no data on blindness or loss of color vision. General adverse events such as hypertension, thromboembolic events, and death were reported in four trials.

\section{Methodological quality}

Overall, three of the trials, ${ }^{20-22}$ assessors were adequately masked. Intravitreal injections were masked in one ${ }^{23}$ and there was masking for the measurement via OCT and fluorescein angiography in the other. ${ }^{24}$ The fundus assessments were performed by two retina specialists who were aware of the treatment assignment. Study data were collected, interpreted, and analyzed by two other masked investigators. Intentionto-treat analysis was performed in three trials..$^{20,21,23}$

Randomization consisted of varying length of permuted blocks, simple randomization, and applying a dynamic minimization procedure using a stochastic treatment allocation algorithm based on a variance method. The process of randomization was described in one study, ${ }^{24}$ but allocation concealment was not described in any of the trials.

In the Ahmadieh et $\mathrm{al}^{20}$ study, one patient in the control group died during the study period. Cunningham et $\mathrm{al}^{21}$ left out nine patients from the study as a result of one death and eight patient requests. Scott et $\mathrm{al}^{22}$ had two subjects withdraw before completion of the study and their overall visit completion rate was $93 \%$. Paccola et $\mathrm{al}^{24}$ reported that two subjects missed two consecutive treatments, while Soheilian et $\mathrm{al}^{23}$ reported no losses to follow-up.

\section{Results}

Meta-analysis of data was only possible for three trials. ${ }^{20,21,23}$ We were unable to use data from one trial, ${ }^{22}$ as results were expressed in medians and interquartile ranges. The authors have been contacted for their raw data, including means and standard deviations. This trial will be added to the metaanalysis once appropriate results obtained.

The study by Paccola et $\mathrm{al}^{24}$ was not included in the metaanalysis as this study compared intravitreal bevacizumab to intravitreal triamcinolone instead of a control or standard therapy such as laser. The study by Paccola et $\mathrm{al}^{24}$ has been included in the qualitative analysis of anti-VEGF.

It should be noted that with respect to the forest plots reported in this review, for outcomes such as gain in visual acuity and CMT, effect estimates to the right of the vertical line favor test treatment.

Anti-VEGF treatments were shown to have a benefit in improving BCVA. For instance, Cunningham et $\mathrm{al}^{21}$ compared three differing doses of intravitreal pegaptanib compared to a control injection. Three forests plots each with a differing dose of pegaptanib showed a consistent benefit with the intervention on visual acuity. Using pegaptanib $0.3 \mathrm{mg}$ in the meta-analysis, the mean change in visual acuity was -0.17 ( $95 \%$ confidence interval $[\mathrm{CI}]:-0.23,-0.10)$. A similar treatment effect was seen using $1 \mathrm{mg}$ and $3 \mathrm{mg}$ of pegaptanib with a mean difference of -0.17 (95\% CI: $-0.23,-0.10)$ and -0.14 (95\% CI: $-0.20,-0.07$ ), respectively (Figures 2-4).

Anti-VEGF therapy has a benefit on CMT. Cunningham et $\mathrm{al}^{21}$ compared three differing doses of intravitreal pegaptanib to a control injection. Three forests plots, each demonstrating a differing dose of pegaptanib, showed a consistent benefit of the intervention on CMT. Using pegaptanib $0.3 \mathrm{mg}$ in the meta-analysis, the mean change on CMT was found to be -84.69 (95\% CI: 117.09, -52.30). A similar treatment effect was seen using $1 \mathrm{mg}$ and $3 \mathrm{mg}$ of IVP with a mean difference $-84.69(95 \% \mathrm{CI}:-117.09,-52.30)$ and $-72.47(95 \%$ CI: -106.67, -38.27) in evidence (Figures 5-7).

Combined anti-VEGF with intravitreal triamcinolone was shown to benefit both visual acuity and central macular thickness compared to the control. Mean difference for visual acuity

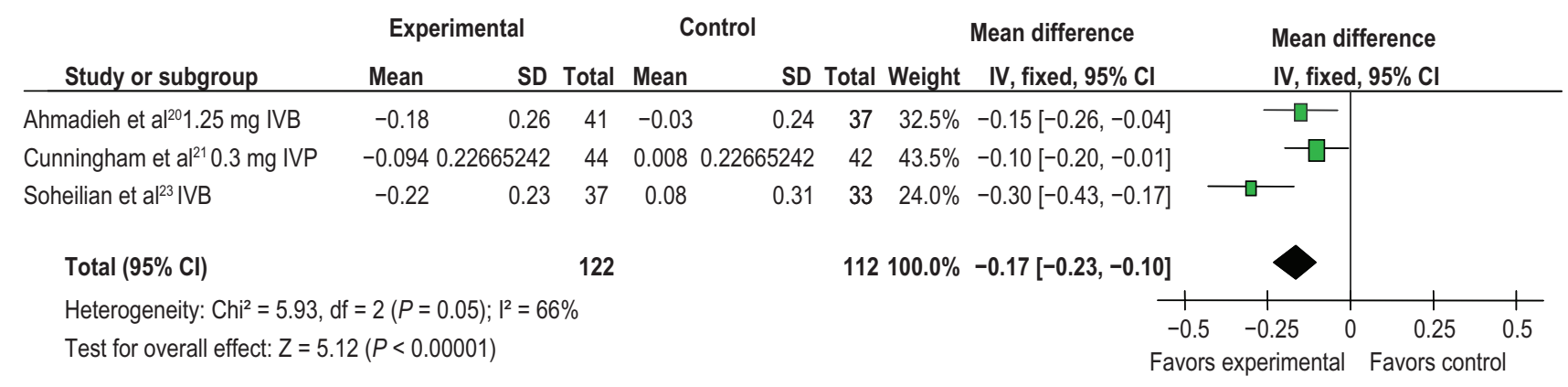

Figure 2 Effect of best corrected visual acuity using anti-VEGF ( $0.3 \mathrm{mg}$ of pegaptanib in one study).

Abbreviations: anti-VEGF, anti-vascular endothelial growth factor; SD, standard deviation; Cl, confidence interval; IVB, intravitreal bevacizumab; IVP, intravitreal pegaptanib. 


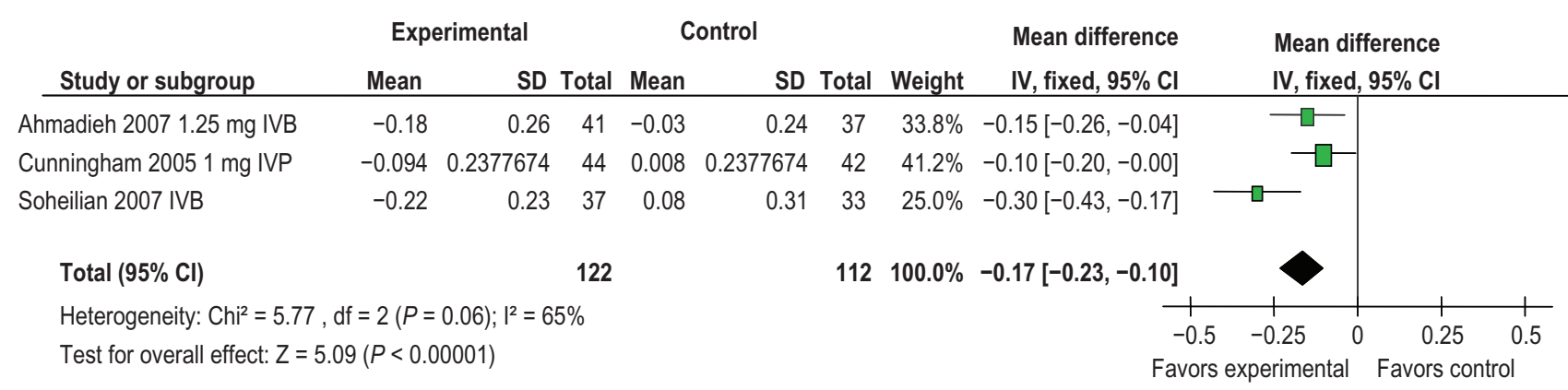

Figure 3 Effect of best-corrected visual acuity using anti-VEGF (I mg of pegaptanib in one study).

Abbreviations: $\mathrm{Cl}$, confidence interval; IV, intravenous; IVB, intravitreal bevacizumab; IVP, intravitreal pegaptanib; SD, standard deviation; VEGF, vascular endothelial growth factor.

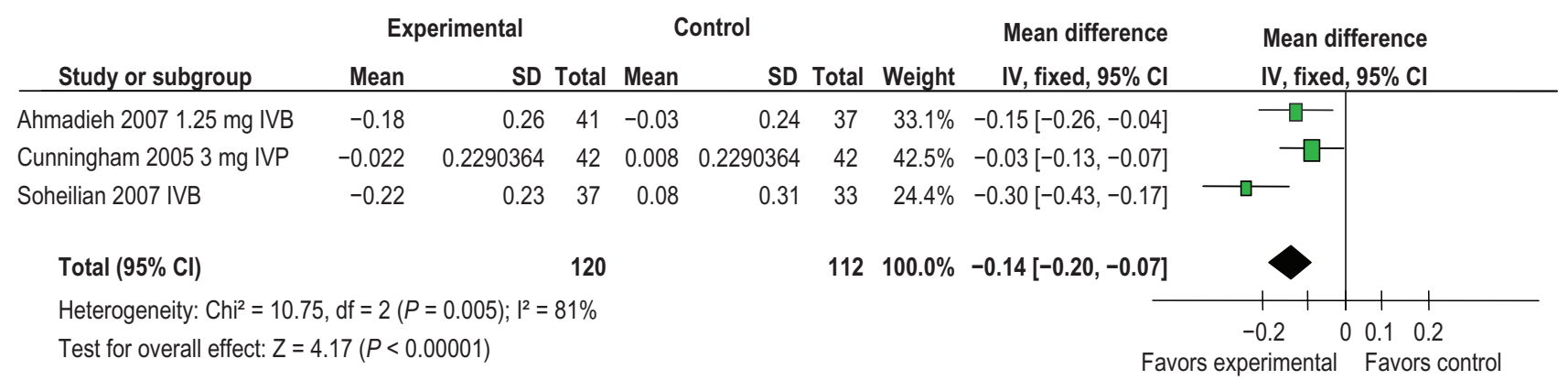

Figure 4 Effect of best-corrected visual acuity using anti-VEGF ( $3 \mathrm{mg}$ of pegaptanib in one study).

Abbreviations: $\mathrm{Cl}$, confidence interval; IV, intravenous; IVB, intravitreal bevacizumab; IVP, intravitreal pegaptanib; SD, standard deviation;VEGF, vascular endothelial growth factor.

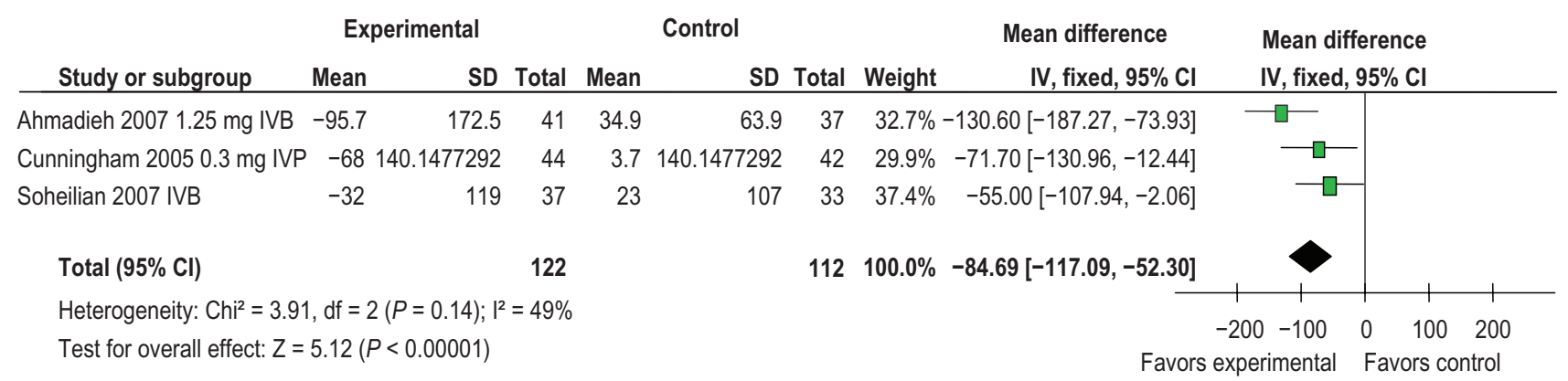

Figure 5 Effect of central macular thickness using anti-VEGF (0.3 mg of pegaptanib in one study).

Abbreviations: $\mathrm{Cl}$, confidence interval; IV, intravenous; IVB, intravitreal bevacizumab; IVP, intravitreal pegaptanib; SD, standard deviation;VEGF, vascular endothelial growth factor.

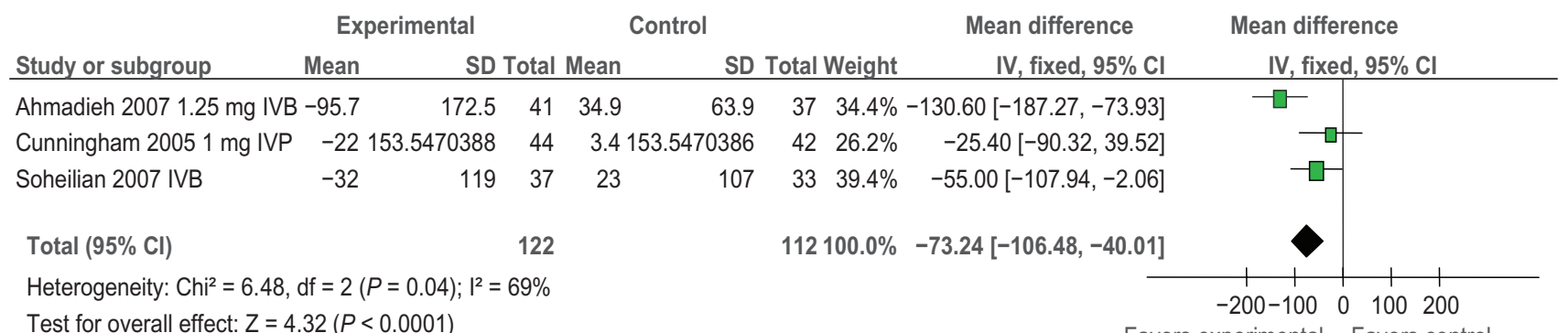

Test for overall effect: $Z=4.32(P<0.0001)$

Favors experimental Favors control

Figure 6 Effect for central macular thickness using anti-VEGF (I mg of pegaptanib in one study).

Abbreviations: $\mathrm{Cl}$, confidence interval; IV, intravenous; IVB, intravitreal bevacizumab; IVP, intravitreal pegaptanib; SD, standard deviation;VEGF, vascular endothelial growth factor. 


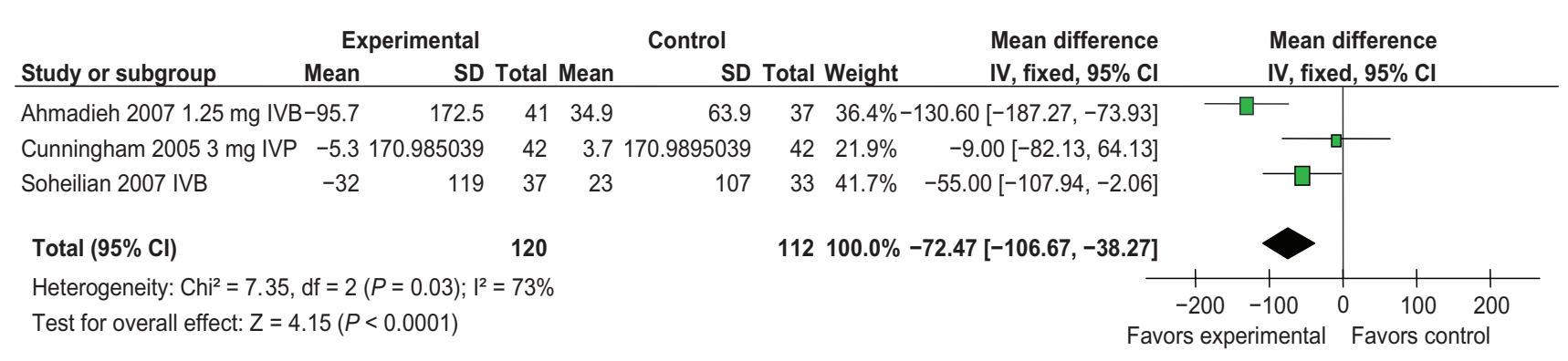

Figure 7 Effect of central macular thickness using anti-VEGF (3 $\mathrm{mg}$ of pegaptanib in one study).

Abbreviations: Cl, confidence interval; IV, intravenous; IVB, intravitreal bevacizumab; IVP, intravitreal pegaptanib; SD, standard deviation; VEGF, vascular endothelial growth factor.

was $-0.19(95 \% \mathrm{CI}:-0.27,-0.11)$ and the CMT mean change was -111.20 (95\% CI: -148.13, -74.28) (Figures 8 and 9).

Scott et $\mathrm{al}^{22}$ revealed a benefit in central macular thickness when using the intervention of $1.25 \mathrm{mg}$ and $2.5 \mathrm{mg}$ of intravitreal bevacizumab compared to the use of laser. There was only a trend towards reduction up to 12 weeks, however. Visual acuity also improved with the two IVB interventions compared to laser at 3 weeks.

There was significant heterogeneity amongst the trials: $65 \%-81 \%$ for the assessment of visual acuity and $49 \%-73 \%$ for CMT. Despite the high $\mathrm{I}_{2}$, results were pooled and reported, as examination of the forest plot indicated that the individual trial results were consistent in the direction of the effect (ie, the mean difference, standard deviation and confidence intervals largely fell on the side of the null line favoring the intervention).

Subgroup analysis and assessment of publication bias could not be preformed due to small study numbers and data set. Meta-regression may be preformed in future to group the differing interventions in the Cunningham ${ }^{21}$ and $\mathrm{Scott}^{22}$ studies compared to a control group once data becomes available.

The pooled trend favors intervention using anti-VEGF to increase visual acuity and encourage CMT changes. Furthermore, trial quality was found to be better among those showing an improvement of BCVA and CMT using anti-VEGF.

\section{Complications}

There were no significant increases in complications reported among the interventions trialed. Complications associated with intravitreal injections, including ocular hypertension, were noted in a few studies. All trials noted to have ocular hypertension comment of successful treatment of increased IOP. Table 5 shows a summary of included study adverse events.

\section{Discussion}

In this systematic review of randomized controlled trials our meta-analysis has shown that anti-VEGF alone or in combination with triamcinolone is effective in the treatment of diabetic macular edema with an improved change in BCVA and CMT. Over with the treatment duration ranging from 12 to 36 weeks the initial analysis has shown a short-term benefit for the intervention and available information of adverse effects does not suggest potentially vision threatening complications with intravitreal injections.

Only the three trials included in the meta-analysis were of good methodological quality. There is no direct evidence comparing different types of anti-VEGF therapies so far published. The Scott et $\mathrm{al}^{22}$ trial was conducted by a pharmaceutical company, thus has potential for bias due to conflict of interest. As mentioned above, we were also unable to use this trial in the meta-analysis due to the presentation of data

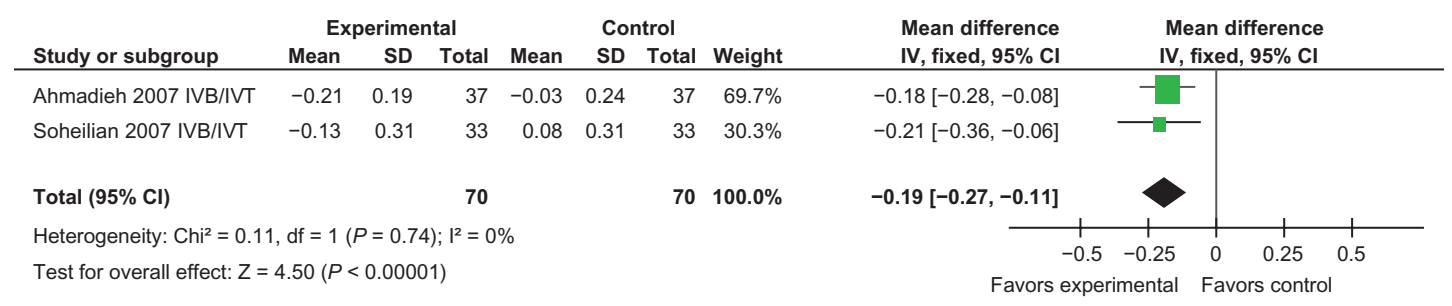

Figure 8 Effect of best-corrected visual acuity using IVB/IVT.

Abbreviations: $\mathrm{Cl}$, confidence interval; IV, intravenous; IVB, intravitreal bevacizumab; IVP, intravitreal pegaptanib; SD, standard deviation; VEGF, vascular endothelial growth factor. 


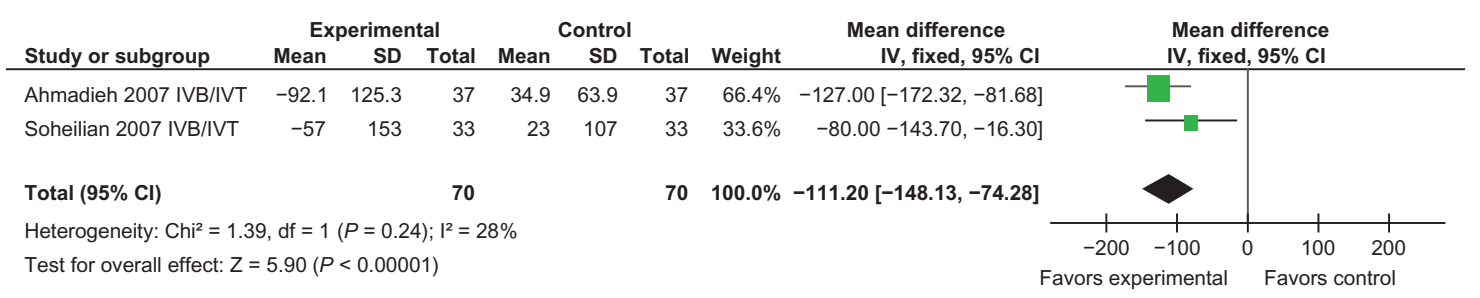

Figure 9 Effect of central macular thickness using IVB/IVT.

Abbreviations: $\mathrm{Cl}$, confidence interval; IV, intravenous; IVB, intravitreal bevacizumab; IVP, intravitreal pegaptanib; SD, standard deviation;VEGF, vascular endothelial growth factor.

using medians and interquartile ranges. Our assumption was that the outcome distributions were skewed.

Cunningham et a ${ }^{21}$ used three differing doses of IVP compared to a control. We were unable to scrutinize these interventions together in the same analysis as the same group of control subjects would have been entered twice. Hence, three separate analysis tables were calculated, each representing a different dose, from this trial. Future meta-regression to pool these groups may be performed.

Paccola et $\mathrm{al}^{24}$ suggest intravitreal triamcinolone has a significant benefit in visual acuity over intravitreal bevacizumab. There was also a significant reduction in CMT in the intravitreal triamcinolone group at week $24(P=0.024)$, with similar results for visual acuity compared with IVB. Analysis of IVB was not performed to control so, although a benefit was anecdotally noted to improve visual acuity and CMT from baseline, this provides no significance statistically.

Subgroup analysis was not performed due to the limited number of studies. Similarly, assessment of publication bias was not performed due to the limited number of studies included in meta-analysis. Future inclusion of more clinical trials is needed for meaningful subgroup analysis including assessment of publications bias.

Quality of life and economic data were not available in any of the included studies making it is difficult to assign meaning to LogMAR changes in visual acuity. However, the trend is favorable towards intervention with regards to improvement of vision and perhaps slowing further progression of disease and its associated morbidity. Reduction in macular thickness, especially in regards to CMT, is considered a mechanism for visual improvement in the treatment of macular edema. Therefore, a corresponding reduction in CMT would be expected if there was a trend towards vision improvement. This is confirmed in the analysis where the intervention using anti-VEGF favored CMT improvement.

As current treatment is directed at stabilizing or reducing vision loss, an important finding is that there were no significant increases in complications among the interventions.
The results were limited by heterogeneity in the included trials. The difference in the intervention doses and duration of treatment contributed to this. A strength within the studies is that the exact definition and measurement of outcomes were fairly consistent and our pooled results should not be biased due to misclassification. The limited data for the Scott et al ${ }^{22}$ study made it difficult to quantify the trial results concerning the effect of the intervention in a meaningful way.

Overall, most studies show a promising trend towards benefits in visual acuity and central macular thicken with use of anti-VEGF. Excluded studies continue to confirm this trend for most patients, although subgroups of patients with DME with ischemia were found to have a negative outcome. ${ }^{15}$

\section{Conclusion}

Strict glycemic and blood pressure control are still the hallmarks of prevention and progression of diabetic macular edema. The ETDRS brought laser therapy into consideration for mainstream use for diabetic macular edema with some modest benefit to visual acuity evidenced. ${ }^{6}$

Anti-VEGF was associated with an improvement in visual acuity and CMT in our analysis. Trials studied were all of a short duration and there were no long-term follow-up studies found. There was no evidence found comparing the different types of anti-VEGF. The use of anti-VEGF is promising to improve visual acuity and CMT caused by diabetic macular edema. Further trials are being conducted, at present, with ongoing follow-up studies to assist in determining overall long-term benefit. The Appendix outlines some of the characteristics of these ongoing studies.

\section{Implications for future research}

The five studies in included in our report dealt primarily with persistent or refractory diabetic macular edema and raised issues including the duration of intervention, dosage, timing of repeats and follow-up, and the role of combination therapy with intravitreal triamcinolone in relationship to control or laser therapy. The question arises whether treatment 
should be reserved for just this chronic or refractory group of patients or introduced during earlier stages of diabetic macular edema. Economic and quality of life data need to be considered, as well as further quantitative analysis of overall effects of complications. Long-term, follow-up studies with considerations for adverse effects need to be quantified and documented to provide a better understanding of risk benefits for patients with diabetic macular edema.

\section{Acknowledgments}

The contributions of Dr Benjamin Tang of the University of Sydney, School of Public Health are gratefully acknowledged.

\section{Disclosure}

The authors report no conflicts of interest in this work.

\section{References}

1. Nguyen QD, Tatlipinar S, Shah SM, et al. Vascular endothelial growth factor is a critical stimulus for diabetic macular edema. Am J Ophthalmol. 2006;142:961-969.

2. Arevalo JF, Fromow-Guerra J, Quiroz-Mercado H, et al. Primary intravitreal bevacizumab (Avastin ${ }^{\circledR}$ ) for diabetic macular edema: results from the Pan-American Collaborative Retina Study Group at 6-month follow-up. Ophthalmology. 2007;114:743-750.

3. Grover D, Li TJ, Chong CCW. Intravitreal steroids for macular edema in diabetes. Cochrane Database Syst Rev. 2008;(1):CD005656.

4. Mitchell P, Foran S. Guidelines for the Management of Diabetic Retinopathy. The National Health and Medical Research Council (NHMRC). Canberra, Australia: Australian Diabetes Society for the Department of Health and Aging. 2008.

5. Soliman W, Vinten M, Sander B, et al. Optical coherence tomography and vessel diameter changes after intravitreal bevacizumab in diabetic macular edema. Acta Opthalmol. 2008;86:365-371.

6. Fong DS, Ferris III FL, Davis MD, Chew EY. The Early Treatment of Diabetic Retinopathy Study Research Group. Photocoagulation for diabetic macular edema. ETDRS Report No 1. Arch Ophthalmol. 1985;103:1644-1652.

7. Thomas D, Bunce C, Moorman C, Laidlaw DA. A randomised controlled feasibility trial of vitrectomy versus laser for diabetic macular edema. Br J Ophthalmol. 2005;89:81-86.

8. Jain RK. The VEGF ligand and angiogenesis. Science. 2005;307: $58-62$.

9. Aiello LP, Avery RL, Arrigg PG, et al. Vascular endothelial growth factor in ocular fluid of patients with diabetic retinopathy and other retinal disorders . N Eng J Med. 1994;331:1480-1487.

10. Emerson MV, Lauer AK. Emerging therapies for the treatment of neovascular age-related macular degeneration and diabetic macular edema. BioDrugs. 2007;21:245-257.

11. Ng EW, Shima DT, Calias P, Cunningham ET Jr, Guyer DR. Pegaptanib, a targeted anti-VEGF aptamer for ocular vascular disease. Nat Rev Drug Discov. 2006;5:123-132.

12. Spaide RF, FisherYL. Intravitreal bevacizumab (Avastin $\left.{ }^{\circledR}\right)$ treatment of proliferative diabetic retinopathy complicated by vitreous hemorrhage. Retina. 2006;26:275-278.

13. Homsi J, Daud AI. Spectrum of activity and mechanism of action of VEGF/PDGF inhibitors. Cance Control. 2007;14:285-294.

14. Vedula SS, krystolik M. Antiangiogenic therapy with anti-vascular endothelial growth factor modalities for neovascular age-related macular degeneration. Cochrane Database Syst Rev. 2008;2:21-62. 
15. Chung EJ, Roh MI, Kwon OW, Koh HJ. Effects of macular ischemia on the outcome of intravitreal bevacizumab therapy for diabetic macular edema. Retina. 2008;28:957-963.

16. US National Institute of Health Clinical Trials register. Available from: www.clinicaltrials.gov. Accessed Jan 10, 2010.

17. Current Controlled Trials register. Available from: http://www. controlled-trials.com. Accessed Jan 10, 2010.

18. The National Research Register (NRR). National Institute of Health Research, National Health System. Available from: www.nrr.nhs.uk.

19. Cochrane Handbook for Systematic Reviews of Interventions 6.7. San Francisco, CA: The Cochrane Collaboration, 2006.

20. Ahmadieh H, Ramezani A, Shoeibi N, et al. Intravitreal bevacizumab with or without triamcinolone for refractory diabetic macular edema; a placebo-controlled, randomized clinical trial. Graefes Arch Clin Exp Ophthalmol. 2008;246:483-489.

21. Cunningham ET Jr, Adamis AP, Altaweel M, et al. Macugen diabetic retinopathy study group. A phase II randomized double-masked trial of pegaptanib, an anti-vascular endothelial growth factor aptamer, for diabetic macular edema. Ophthalmology. 2005;112:1747-1757.

22. Scott IU, Bressler NM, Bressler SB, et al. Diabetic retinopathy clinical research network study group. Agreement between clinician and reading center gradings of diabetic retinopathy severity level at baseline in a phase 2 study of intravitreal bevacizumab for diabetic macular edema. Retina. 2008;28:36-40.

23. Soheilian M, Ramezani A, Bijanzadeh B, et al. Intravitreal bevacizumab $\left(\right.$ Avastin $\left.^{\circledR}\right)$ injection alone or combined with triamcinolone versus macular photocoagulation as primary treatment of diabetic macular edema. Retina. 2007;27:1187-1195.

24. Paccola L, Costa RA, Folgosa MS, Barbosa JC, Scott IU, Jorge R. Intravitreal triamcinolone versus bevacizumab for treatment of refractory diabetic macular edema (IBEME study). Br J Ophthalmol. 2008;92:76-80.
25. Rizzo S, Genovesi-Ebert F, Di Bartolo E, Vento A, Miniaci S, Williams G. Injection of intravitreal bevacizumab (Avastin ${ }^{\circledR}$ ) as a preoperative adjunct before vitrectomy surgery in the treatment of severe proliferative diabetic retinopathy (PDR). Graefes Arch Clini Exp Ophthalmol. 2008;246:837-842.

26. Nguyen QD, Tatlipinar S, Shah SM, et al. Vascular endothelial growth factor is a critical stimulus for diabetic macular edema. Am J Ophthalmol. 2006;142:961-969.

27. Arevalo JF, Fromow-Guerra J, Quiroz-Mercado H, et al. Pan-American Collaborative Retina Study Group. Primary intravitreal bevacizumab $\left(\right.$ Avastin $^{\circledR}$ ) for diabetic macular edema: results from the Pan-American Collaborative Retina Study Group at 6-month follow-up. Multicenter Study. Ophthalmology. 2007;114:743-750.

28. Byeon SH, Kwon YA, Oh HS, Kim M, Kwon OW. Short-term results of intravitreal bevacizumab for macular edema with retinal vein obstruction and diabetic macular edema. J Ocul Pharmacol Ther. 2007;23:387-394.

29. Yanyali A, Aytug B, Horozoglu F, Nohutcu AF. Bevacizumab (Avastin $\left.{ }^{(}\right)$ for diabetic macular edema in previously vitrectomized eyes. Am J of Ophthalmol. 2007;144:124-126.

30. Shimura M, Nakazawa T, Yasuda K, et al. Comparative therapy evaluation of intravitreal bevacizumab and triamcinolone acetonide on persistent diffuse diabetic macular edema. Am J Ophthalmol. 2008;145:854-861.

31. Haritoglou C, Kook D, Neubauer A, et al. Intravitreal bevacizumab $\left(\right.$ Avastin $\left.^{\circledR}\right)$ therapy for persistent diffuse diabetic macular edema. Retina. 2006;26:999-1005.

32. Chun DW, Heier JS, Topping TM, Duker JS, Bankert JM. A pilot study of multiple intravitreal injections of ranibizumab in patients with centerinvolving clinically significant diabetic macular edema. Ophthalmology. 2006;113:1706-1712.

33. Ferrara N. Vascular endothelial growth factor: basic science and clinical progress. Endocr Rev. 2004;25:581-611. 


\section{Appendix}

\section{Table I Search: Central}

I. diabetic macular edema.mp. [mp=title, original title, abstract, mesh headings, heading words, keyword] 18

2. diabetic macula odema.mp. [mp=title, original title, abstract, mesh headings, heading words, keyword] 0

3. Diabetic Retinopathy.mp. [mp=title, original title, abstract, mesh headings, heading words, keyword] 843

4. macular edema.mp. [mp=title, original title, abstract, mesh headings, heading words, keyword] 53

5. Macular Edema, Cystoid.mp. [mp=title, original title, abstract, mesh headings, heading words, keyword] 74

6. Macular Degeneration.mp. [mp=title, original title, abstract, mesh headings, heading words, keyword] 609

7. (DME or DMO or CME or CSME).mp. [mp=title, original title, abstract, mesh headings, heading words, keyword] 197

8 . 6 or 4 or I or 3 or 7 or 2 or 51620

9. angiogenesis inhibitors.mp. [mp=title, original title, abstract, mesh headings, heading words, keyword] 96

10. angiogenesis inhibitors. $\mathrm{mp}$. [ $\mathrm{mp}=$ title, original title, abstract, mesh headings, heading words, keyword] 96

II. endothelial growth factors.mp. [mp=title, original title, abstract, mesh headings, heading words, keyword] 68

12. vascular endothelial growth factors.mp. [mp=title, original title, abstract, mesh headings, heading words, keyword] 65

13. (macugen $\$$ or pegaptanib $\$$ or lucentis $\$$ or rhufab $\$$ or ranibizumab $\$$ or bevacizumab\$).tw. 120

14. (anti adj2 VEGF\$).tw.

15. (endothelial adj2 growth adj2 factor\$).tw. 265

16. (macula $\$$ adj2 swell\$).tw. I

17. 11 or 13 or 10 or 9 or 12 or 15 or 14414

|8. 8 and $177 \mid$

19. from 18 keep 10, 33, 45, 47, 51, 53-54... 9
Table 2 Search: Medline ${ }^{\circledR}$

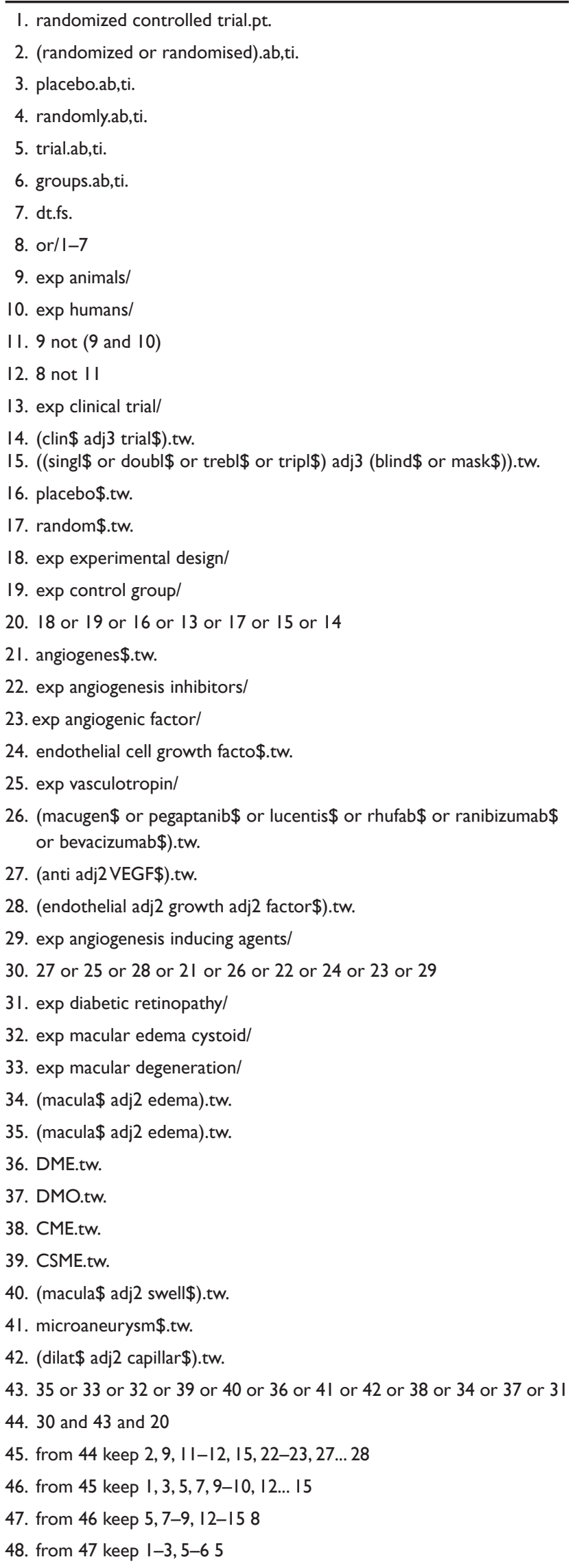




\section{Table 3 Search: Embase ${ }^{\circledR}$}

I. randomized controlled trial.pt.

2. (randomized or randomised).ab,ti.

3. placebo.ab,ti.

4. randomly.ab,ti.

5. trial.ab,ti.

6. groups.ab,ti.

7. dt.fs.

8. or/I-7

9. exp animals/

10. exp humans/

11.9 not (9 and 10)

I2. 8 not II

13. clinical trial.pt

14. cluster trial

15. exp control group

16. double blind\$.tw

17. single blind $\$ . t w$

18. (blind $\$$ or mask $\$$ ).tw

19. exp cross over

20. exp comparative study

21 . prospective $\$$.tw

22. or $|3-2|$

23. or $12-22$

24 . angiogenes $\$$.tw.

25. exp angiogenesis inhibitors/

26. exp angiogenic factor/

27. endothelial cell growth facto $\$ . t w$.

28. exp vasculotropin/

29. (macugen $\$$ or pegaptanib $\$$ or lucentis $\$$ or rhufab $\$$ or ranibizumab $\$$ or bevacizumab\$).tw.

30. (anti adj2 VEGF\$).tw.

31. (endothelial adj2 growth adj2 factor\$).tw.

32. exp angiogenesis inducing agents/

33. or $24-32$

34. exp diabetic retinopathy/

35. exp macular edema cystoid/

36. exp macular degeneration/

37. (macula $\$$ adj2 edema).tw.

38. (macula $\$$ adj2 edema).tw.

39. DME.tw.

40. DMO.tw.

4I. CME.tw.

42. CSME.tw.

43. (macula $\$$ adj2 swell\$).tw.

44. microaneurysm $\$ . t w$.

45. (dilat\$ adj2 capillar\$).tw.

46. or $34-45$

47.23 and 33 and 46

\section{Characteristics of included studies} Ahmadieh et $\mathrm{al}^{20}$

Title: Intravitreal bevacizumab with or without triamcinolone for refractory diabetic macular edema; a placebo-controlled, randomized clinical trial

Methods: Study design: RCT

Method of randomization: Using computer generated random lists a random block permutation was performed with block lengths varying randomly $(3,6)$

Number of randomized: $115 \mathrm{DME}$ eyes (41 eyes in anti IVB arm, 37 eyes in the IVB/IVT arm and 37 eyes in the control arm.)

Method of allocation concealment: no data

Outcome assessor masking: Adequately masked

Study duration: 24 weeks

Losses to follow-up: One patient died in the control group during the study period.

Intention to treat analysis: performed

Participants: Inclusion criteria: Clinically significant macular edema unresponsive to previous macular laser with the last session being more than three months prior.

Exclusion criteria: visual acuity $>20 / 40$, history of cataract surgery within the past 6 months, prior intraocular injection or vitrectomy, glaucoma or ocular hypertension, Proliferative diabetic retinopathy with high risk characteristics, vitreous hemorrhage, significant media opacity and presence and traction on the macula. Monocular patients, pregnant patients and those with a serum creatinine of $>3 \mathrm{mg} / 100$ were excluded.

Type of DME: CSME

Prior laser treatment: All participants

Age: $59.7 \pm 8.3$

Comparability of baseline characteristics: groups were matched for age, sex, baseline visual acuity, hypertension, smoking history, stage of diabetic retinopathy, number of previous laser sessions at the macula and history of PRP $(P>0.05)$ Treatment group were not matched for CMT which was lower in the control group compared to the other two groups $(P<0.05)$.

Interventions: Test intervention: IVB $1.25 \mathrm{mg}$

Test intervention: IVB/IVT (1.25 mg/2 mg)

Control: needleless syringe placed against conjunctiva and sclera. Three injections performed at 6-week intervals.

Outcomes: Primary outcome: change in CMT compared to baseline.

Measurement of primary outcome: CMT by OCT 
Secondary outcome: Change in best corrected LogMAR visual acuity, IOP rise, cataract progression, intraocular inflammation and other serious adverse effects.

Measurement of secondary outcome: VA by ETDRS chart, IOP measure by application tonometer.

Notes: Data source: published data

Funding source: Nonindustry funded

Country: Iran

\section{Cunningham et $\mathrm{al}^{21}$}

Title: A Phase II randomized double-masked trial of pegaptanib, an antivascular endothelial growth factor aptamer, for diabetic macular edema

Methods: Study design: RCT

Method of randomization: Dynamic minimization procedure using a stochastic treatment allocation algorithm based on variance method.

Number of randomized: 172 eyes 44 eyes in the $0.3 \mathrm{mg}$ IVP arm, 44 eyes in the $1 \mathrm{mg}$ IVP arm, 42 eyes in the $3 \mathrm{mg}$ IVP arm and 42 eyes in the sham arm.

Method of allocation concealment: no data

Outcome assessor masking: Adequately masked

Losses to follow-up: 9 patients discontinued from the study ( 1 death and 8 by request)

Intention to treat analysis: performed

Participants: Inclusion criteria: Demonstrated to have macular edema involving the center of the macula demonstrated by OCT with corresponding leakage from micro aneurysms, retinal telangiectatisis, or both on fluorescein angiography. Best-corrected visual acuity (Snellen equivalent) 20/50-20/320. IOP less than $23 \mathrm{mmHg}$ and those patients in which focal photocoagulation could be delayed for at least 16 weeks safely.

Exclusion criteria: History of panretinal or focal photocoagulation within the previous 6 months. Any abnormality thought to confound VA assessments or fundus photography including cataracts, vitreoretinal traction within 1 disc diameter of the fovea confirmed either clinically or on OCT, vitreous incarceration in a previous wound or incision, any retinal vein occlusion involving the macula and atrophy/ scarring/fibrosis or hard exudates involving the center of the macula that would preclude improvement in VA. History of intraocular surgery within 12 months of the study, active ocular infection, serious allergies to fluorescein dye. GHb levels of $>13 \%, 2$ episodes of ketoacidosis within 1 year, severe cardiac disease, clinical significant peripheral vascular disease, uncontrolled hypertension, stroke within 1 year and previous therapeutic radiation to eye, head, or neck.
Types of DME: Focal and diffuse

Prior laser treatment: Some participants, at least 6 months previously

Age: (0.3 mg) 61.9 \pm 10.0; (1 mg) 62.8 \pm 10.1; (3 mg) 61.3 \pm 9.8; (sham) $64.0 \pm 9.3$

Intervention: Test intervention: $0.3 \mathrm{mg}$ of IVP, $1 \mathrm{mg}$ IVP, $3 \mathrm{mg}$ IVP

Control intervention: Sham: needleless syringe pressed against the conjunctiva and sclera

Repeat treatments: injections were given at entry, week 6 , and week 12 for a minimum of three injections. Additional injections were administered every 6 weeks up to week 30 .

Outcomes: Primary outcome: Best-corrected visual acuity and change in CMT compared to baseline.

Measurement of primary outcome: ETDRS chart for VA and OCT for CMT

Secondary outcome: Presence of improvement via fluorescein angiography, IOP, safety endpoints included all adverse events. Vital signs and biochemical analysis of blood and urine performed.

Measurement of secondary outcome: Fluorescein angiography, IOP measure by application tonometer, blood and urine analysis.

Data source: published data

Funding source: Sponsored by Eyetech Pharmaceuticals. Inc New York, New York, and Pfizer Inc ${ }^{\circledR}$, New York, New York Country: United States of America

\section{Scott et $\mathrm{al}^{22}$}

Title: A Phase II randomized clinical trial of intravitreal bevacizumab for diabetic macular edema

Methods: Study design: RCT

Method of randomization: Simple randomization

Number of randomized: 109 eyes included. 19 eyes in the laser group, 22 in the 1.25 IVB group, 24 eyes in the $2.5 \mathrm{mg}$ IVB group, 22 eyes in the $1.25 \mathrm{mg}$ IVB at baseline group and 22 eyes in the $1.25 \mathrm{mg}$ IVB plus laser group.

Method of allocation concealment: No data

Outcome assessor masking: Adequately masked

Losses to follow-up: 2 subjects withdraw before completion. Overall visit completion rate was $93 \%$.

Intention to treat analysis: not performed

Participants: Inclusion criteria: Patients were at least over 18 years of age. Best-corrected electronic ETDRS VA score $>24$ (20/320 or better) and $<78$ (20/32 or worse), defined retinal thickening due to DME involving the center of the macula based on clinical examination. OCT central subfield 
$>275 \mu \mathrm{m}$ and no history of treatment for DME at any time within the prior 3 months.

Exclusion criteria: Only one eye included in the study. Type of DME: retinal thickening due to DME involving the center of the macula

Prior laser treatment: no laser treatment within 3 months of study starting time.

Age: 65

Intervention: Test intervention: $1.25 \mathrm{mg}$ of IVB at baseline and at 6 weeks; $2.5 \mathrm{mg}$ of IVB at baseline and at 6 weeks; $1.25 \mathrm{mg}$ of IVB at baseline; $1.25 \mathrm{mg}$ of IVB at baseline and 6 weeks plus photocoagulation at 3 weeks.

Control intervention: Focal photocoagulation at baseline

Outcomes: Primary outcome: best-corrected visual acuity, CMT

Measurement of primary outcome: ETDRS chart for VA and OCT for CMT

Secondary outcome: Adverse effects; endophthalmitis, IOP, cardiac dysfunction, BP

Measurement of secondary outcome: clinical examination and IOP measure by application tonometer

Data source: published data

Funding source: National Eye Institute, Bethesda, Maryland and by a grant from the Juvenile Diabetes Research Foundation International, New York, New York

Country: United States of America

\section{Soheilian et $\mathrm{a}^{23}$}

Title: Intravitreal bevacizumab (Avastin) injection alone or combined with triamcinolone versus macular photocoagulation as primary treatment of diabetic macular edema

Methods: Study design: RCT

Method of randomization: Random block permutation method performed using computer generated random list. Block length varied randomly $(6,12)$.

Number of randomized: 103 eyes 37 eyes in the IVB arm, 33 eyes in the IVB/IVT arm and 33 eyes in the MPC arm.

Method of allocation concealment: No data

Outcome assessor masking: Intravitreal injections were masked.

Losses to follow-up: nil

Intention to treat analysis: performed

Participants: Inclusion criteria: clinically significant macular edema based on the ETDRS definitions.

Exclusion criteria: Previous panretinal or focal laser photocoagulation, prior intraocular injection or operation, history of glaucoma or ocular hypertension, visual acuity of $20 / 40$ or better or worse than $20 / 320$, presence or iris neovascularization, significant media opacity and high risk or active diabetic retinopathy. Monocularity, pregnancy and creatinine level $>3 \mathrm{mg} / \mathrm{dL}$ were excluded.

Types of DME: focal and diffuse

Prior laser treatment: no

Age: $62.4 \pm 6.1$

Intervention: Test intervention: $1.25 \mathrm{mg}$ IVB; $1.25 \mathrm{mg}$ IVT; 2 mg IVT

Control intervention: macular laser photocoagulation

Repeat treatment: no

Outcomes: Primary outcome: Change in best-corrected visual acuity (LogMAR), CMT changes

Measurement of primary outcome: VA by ETDRS chart, OCT for CMT

Secondary outcome: Changes in fluorescein angiography leakage, ocular hypertension, anterior chamber reaction and lens opacity progression.

Measurement of secondary outcome: leakage assessed by fluorescein angiography, IOP measure by application tonometer and clinical examination performed.

Data source: published data

Funding source: Ophthalmic Research Centre of Shaeed Beheshti Medical University Tehran Iran

Country: Iran

\section{Paccola et $\mathrm{a}^{24}$}

Title: Intravitreal triamcinolone versus bevacizumab for treatment of refractory diabetic macular edema (IBEME) study

Methods: Study design: RCT

Method of randomization: no data

Number of randomized: 28 eyes randomized 13 eyes IVB arm 13 eyes in IVT arm two losses

Method of allocation concealment: no data

Outcome assessor masking: Adequately masked for OCT, fluorescein angiography and fundus assessment were performed by two retina specialists who were aware of the treatment assignment. Study data were collected and interpreted and analyzed by two other masked investigators.

Losses to follow-up: two patients missed two consecutive treatments

Intention to treat analysis: not performed

Participants: Inclusion criteria: Refractory DME (defined herein as the presence of clinically significant macula edema as per ETDRS criteria by biomicroscopic evaluation which had persisted despite at least one session of macula laser photocoagulation performed at least 3 months earlier). 
Patients to have diffuse fluorescein leakage involving the foveal center and most of the macula area. A (LogMAR) ETDRS BCVA of 0.3 (20/40) or worse and a CMT greater than $300 \mu \mathrm{m}$ on OCT.

Exclusion criteria: Aphakic or pseudophakic eyes, glycosylated hemoglobin (HBA1c) level above 10\%, history of glaucoma or ocular hypertension defined by a pressure higher than $22 \mathrm{mmHg}$. An ocular condition other than diabetes that may effect or alter the visual acuity during the study example retinal vein occlusion, uveitis, neovascular glaucoma or other inflammatory disease. Patients with a history of a thromboembolic event (MI or CVA) or major surgery within the past 6 months were excluded. Controlled HT, known coagulation abnormalities or current use of anticoagulative medications other than aspirin or any condition affecting follow-up or documentation.

Types of DME: refractory diffuse or focal DME

Prior laser treatment: all participants

Age: 65.58 (8.44) IVB, 67.08 (4.67) IVT

Intervention: Test intervention: $1.5 \mathrm{mg}$ IVB

Control intervention: $4 \mathrm{mg}$ of IVT

Repeat treatment: no

Outcomes: Primary outcome: change in best visual acuity (LogMAR), CMT changes.

Measurement of primary outcome: VA by ETDRS chart, OCT for CMT

Secondary outcome: IOP, cataract progression, and systemic adverse effects.

Measurement of secondary outcome: IOP measure by application tonometer, dilated slit lamp biomicroscopic examination, indirect fundus examination, color fundus photography, and fluorescein angiography performed.

Data source: published data

Funding source: Conselho Nacional de Desenvolvimento Cientiffico e Tecnologico (CNPq)

Country: Brazil

\section{Characteristics of excluded studies}

Rizzo et $\mathrm{al}^{25}$

Title: Injection of intravitreal bevacizumab (Avastin) as a preoperative adjunct before vitrectomy surgery in the treatment of severe Proliferative diabetic retinopathy (PDR)

Methods: Study design: RCT

Method of randomization: Random numbers list used in order to assign group 1 or 2 .

Number randomized: 22 patients 11 eyes with preoperative IVB 11 patients no intervention.
Method of allocation concealment: no data

Outcome assessor masking: no

Losses to follow-up: none

Intention to treat analysis: performed

Participants: Inclusion criteria: tractional retinal detachment, tractional-rhegmatogenous retinal detachment or tractional detachment complicated with vitreous hemorrhage.

Exclusion criteria: History of vitrectomy in the study eye, history of thromoboembolic events (including MI or CVA), major surgery within the last 3 months or planned in the next 28 days, uncontrolled hypertension, known coagulation abnormalities or currently or use of anticoagulative medication other than aspirin.

Types of DME: not given; patient diagnosed with proliferative diabetic retinopathy.

Prior laser treatment: unknown

Age: 52

Intervention test: IVB $1.25 \mathrm{mg}$

Control intervention: No intervention

Outcomes: Primary outcome: feasibility of the surgery

Measurement of primary outcome: duration of surgery, average number of tools exchanged during surgery: number of blunt or sharp dissections, intraoperative bleeding, frequency of endodiatermy, and number of intraoperative breaks.

Secondary outcome: visual and anatomically outcome at 6 months

Measurement of secondary outcome: ophthalmological exam including BCVA slit-lamp examination, retinal fluorescein angiography, and blood pressure measurement.

Data source: published data

Funding source: not given

Country: Italy

Reason for exclusion: Participant has severe proliferative diabetic retinopathy. No mention of diabetic macular edema. Patient's further intervention includes vitrectomy surgery.

\section{Nguyen et $\mathrm{al}^{26}$}

Title: Vascular endothelial growth factor is a critical stimulus for diabetic macular edema

Methods: Study design: nonrandomized clinical trial

Method of randomization: not randomized

Number of patients: 10

Method of allocation concealment: none

Outcome assessor masking: none

Losses to follow-up: none

Intention to treat analysis: N/A 
Participants: Inclusion criteria: 18 years or older, VA between 20/40 and 20/320, a foveal thickness by OCT of $250 \mu \mathrm{m}$ or greater, serum $\mathrm{HbA} 1 \mathrm{c}>6 \%$ for 12 months before randomization, no potential contributing causes to reduce visual acuity other than DME and a reasonable expectation that photocoagulation would not be required for the next six months.

Prior laser treatment: not specified

Age: 60

Intervention: Test intervention: IVR $0.5 \mathrm{mg}$ at baseline, 1, 2, 4 and 6 months.

Control intervention: no control

Outcomes: Primary outcome: Foveal thickness

Measurement of primary outcome: OCT

Secondary outcome: Macular volume and VA

Measurement of secondary outcome: macular volume measured by OCT and VA that was measured by protocol of the ETDRS.

Data source: published data

Funding source: The Wilmer Eye Institute, The Johns Hopkins University School of Medicine, and Baltimore Maryland. Innovative Grant Award from the juvenile Diabetes

Research Foundation by a scholarship from the scientific and technological Research Council of Turkey (S.T) and by a K23 Career Development Award from the National Eye Institute.

Drug provided by Genentech, Inc.

Country: United States of America

Reason for exclusion: Nonrandomized clinical trial.

\section{Arevalo et $\mathrm{al}^{27}$}

Title: Primary intravitreal bevacizumab (Avastin) for diabetic macular edema. Results from the Pan-American Collaborative Retina Study Group at 6 month follow-up

Methods: Study design: retrospective review

Method of randomization: N/A

Number of randomization: N/A

Number studied: 110 eyes reviewed, 78 eyes with follow-up of 6 months

\section{Method of allocation concealment: N/A}

Outcome assessor masking: N/A

Losses to follow-up: 32 eyes with less than 6-month follow-up

Intention to treat analysis: N/A

Participants: Inclusion criteria: Patients treated with at least one injection of IVB $1.25 \mathrm{mg}$ or $2.5 \mathrm{mg}$.

Exclusion criteria: Patients with DME previously treated with laser photocoagulation or IVT. Patients with macular ischemia and the presence of an epiretinal membrane or vitreomacular traction syndrome. Patients with uncontrolled hypertension and recent thromboembolic event.

Types of DME: all

Prior laser treatment: no

Age: $59.7 \pm 9.3$

Intervention: Test intervention: IVB $1.25 \mathrm{mg}$ and $2.5 \mathrm{mg}$ Control intervention: N/A

Repeat treatment: second injection

Outcomes: Primary outcome: changes in BCVA, CMT, and angioscopy changes

Measurement of primary outcome: ETDRS VA, OCT, fluorescein angiography.

Secondary outcome: adverse effects

Measurement of secondary outcome: ophthalmoscopic examination, fluorescein angiography, IOP measure by application tonometer.

Data source: published data

Funding source: Supported in part by the Arevalo-Coutinho Foundation for Research in Ophthalmology, Caracas, Venezuela.

Country: Venezuela, Mexico, Costa Rica, and Brazil

Reason for exclusion: nonrandomized clinical trial.

\section{Chung et al $^{15}$}

Title: Effects of macular ischemia on the outcome of intravitreal bevacizumab therapy for diabetic macular edema

Methods: Study design: retrospective review

Method of randomization: N/A

Number of randomization: N/A

Number studied: 59 eyes of 53 patients

Method of allocation concealment: N/A

Outcome assessor masking: adequate masking

Losses to follow-up: N/A

Intention to treat analysis: N/A

Participants: Inclusion criteria: clinically significant DME according to ETDRS study, a minimum follow-up time of 3 months and recent fluorescein angiography within two weeks before treatment. All three criteria needed to be met. Exclusion criteria: intraocular surgery, including cataract extraction, laser treatments including panretinal photocoagulation, posterior capsulotomy, or focal/grid macular photocoagulation, within 6 months before the treatment and the presence of comorbid ocular conditions that might affect VA.

Type of DME: CSME

Prior laser treatment: greater than 6 months previously

Age: ischemic group 65, nonischemic group 63.4 
Intervention: Test intervention: $1.25 \mathrm{mg}$ IVB

Control intervention: $\mathrm{N} / \mathrm{A}$

Repeat treatment: 1 months 3 months

Outcomes: Primary outcome: BCVA and foveal thickness

Measurement of primary outcome: ETDRS scores for VA and OCT for foveal thickness.

Secondary outcome: injection complications, leakage

Measurement of secondary outcome: IOP measure by application tonometer and fluorescein angiography for assessment of leakage.

Data source: published data

Funding source: Department of Ophthalmology, NHIC Ilsan Hospital, Gyounggido, Korea; and The Institute of Vision Research, Department of Ophthalmology, Yonsei University College of Medicine, Seoul, Korea

Country: Korea

Reason for exclusion: nonrandomized clinical trial.

\section{Byeon et $\mathrm{al}^{28}$}

Title: Short-term results of intravitreal bevacizumab for macular edema with retinal vein obstruction and diabetic macular edema

Methods: Study design: retrospective review

Method of randomization: N/A

Number of randomization: N/A

Number studied: 39 eyes studied 25 eyes with DME studied

Method of allocation concealment: N/A

Outcome assessor masking: N/A

Losses to follow-up: N/A

Intention to treat analysis: N/A

Participants: Inclusion criteria: macula edema caused by central (CRVO) or branched retinal vein occlusion (BRVO), or DR, and on the basis of ME severity involving the foveal center and/or a failure to respond to other treatments. Bevacizumab administration commenced at least 3 months after cessation of previous treatments.

Exclusion criteria: Patients with ocular trauma, inflammation, surgery within the previous 3 months, or glaucoma or ocular hypertension were excluded. In addition, patients with uncontrolled hypertension, cerebrovascular accidents or ischemic cardiovascular disease were excluded.

Types of DME: all

Prior laser treatment: greater than 3 months previously

Age: $62.5 \pm 10.7$ in patients with DME

Intervention: Test intervention: $1.25 \mathrm{mg}$ IVB

Control intervention: N/A
Repeat treatment: Yes

Outcomes: Primary outcome: LogMAR visual acuity and central retinal thickness (CRT).

Measurement of primary outcome: ETDRS chart (LogMAR) for VA and OCT for CRT.

Secondary outcome: IOP, changes in macula edema, and leakage.

Measurement of secondary outcome: Measurement of IOP by tonometer, slit-lamp evaluation and fundus examination, and fluorescein angiography

Data source: published data

Funding source: Institute of Vision Research

Department of Ophthalmology Yonsei University College of Medicine 134 Shinchon-Dong Seodaemun-Gu, Seoul Korea

Country: Korea

Reason for exclusion: nonrandomized clinical trial.

Yanyali et $\mathrm{al}^{29}$

Title: Bevacizumab (Avastin) for diabetic macular edema in previously vitrectomized eyes

Methods: Study design: Retrospective, non comparative, interventional case series.

Method of randomization: N/A

Number of randomization: N/A

Number studied: 10 eyes

Method of allocation concealment: N/A

Outcome assessor masking: $\mathrm{N} / \mathrm{A}$

Losses to follow-up: N/A

Intention to treat analysis: N/A

Participants: Inclusion criteria: persistent DME despite prior pars plana vitrectomy with internal limiting membrane removal.

Exclusion criteria: With only focal macular edema attributable to focal leaks from micro aneurysms, treated with macular laser photocoagulation within six months, treated with panretinal photocoagulation within 12 months, with traction retinal detachment, with active neovascularization, with media opacity such as cataract or vitreous hemorrhage, with evidence of epiretinal membrane shown by fundus examination and OCT (Stratus III OCT; Carl Zeiss, Dublin, California, USA), treated with PPV and ILM removal within 12 months, and underwent cataract extraction within six months. Patients with chronic renal failure maintained with renal dialysis were excluded from the study as well.

Types of DME: all

Prior laser treatment: previous 12 months

Age: $62 \pm 8$ 
Intervention: Test intervention: $1.25 \mathrm{mg}$ IVB

Control intervention: $\mathrm{N} / \mathrm{A}$

Repeat treatment: all eyes given 3 injections of IVB

Outcomes: Primary outcome: Foveal thickness and BCVA

Measurement of primary outcome: OCT to measure foveal thickness and ETDRS chart for VA

Secondary outcome: no data

Measurement of secondary outcome: no data

Data source: published data

Funding source: N/A

Country: Turkey

Reason for exclusion: nonrandomized clinical trial. Patient's previous vitrectomy performed.

\section{Shimura et al ${ }^{30}$}

Title: Comparative therapy evaluation of intravitreal bevacizumab and triamcinolone acetonide on persistent diffuse diabetic edema

Methods: Study design: prospective comparative interventional case series

Method of randomization: N/A

Number of randomization: N/A

Number studied: 28 eyes of 14 patients. In each patient one eyes received IVB and the other eyes received IVT.

Method of allocation concealment: N/A

Losses to follow-up: 2 patients dropped out for personal reasons

Intention to treat analysis: not performed

Participants: Inclusion criteria: patients with bilateral DME whose foveal thickness was more than $400 \mu \mathrm{m}$ and who had a visual acuity of resolution (LogMAR) 0.3 in both eyes.

Exclusion criteria: previous therapy for macular edema including grid laser treatment (within 12 months of study start), intravitreal injection of any drugs or vitrectomy surgery.

Types of DME: diffuse and focal

Prior laser treatment: 12 months prior

Age: 65.7

Intervention: Test intervention: One eye $1.25 \mathrm{mg}$ IVB other eye $4 \mathrm{mg}$ IVT

Repeat treatment: No

Outcomes: Primary outcome: BCVA and foveal thickness

Measurement of primary outcome: ETDRS chart for VA and OCT for foveal thickness

Secondary outcome: IOP and diffuse leakage around fovea
Measurement of secondary outcome: leakage assessed by fluorescein angiography

Data source: published data

Funding source: Department of Ophthalmology, NTT East Japan Tohoku Hospital, Sendai, Miyagi, Japan Department of Ophthalmology and Visual Science, Tohoku University Graduate School of Medicine, Sendai, Miyagi, Japan Shiono Eye Center, Sendai, Miyagi, Japan Department of Ophthalmology, Fukushima Medical University, School of Medicine, Fukushima, Japan Department of Ophthalmology, Kagoshima University, Graduate School of Medical and Dental Sciences, Kagoshima, Japan.

Country: Japan

Reason for exclusion: nonrandomized clinical trial.

\section{Haritoglou et $\mathrm{al}^{31}$}

Title: Intravitreal bevacizumab (Avastin) therapy for persistent diffuse diabetic macula edema

Methods: Study design: prospective, consecutive, noncomparative case series

Method of randomization: N/A

Number of randomization: N/A

Number studied: 51 patients 51 eyes studied with IVB

Method of allocation concealment: N/A

Outcome assessor masking: N/A

Losses to follow-up: all patients completed 6 weeks of follow-up; 23 (45\%) completed 12 weeks of follow-up. Sixteen patients $(70 \%)$ had received at least two intravitreal injections

Intention to treat analysis: not performed

Participants: Inclusion criteria: patients with diffuse, clinically significant diabetic macular edema who did not respond to other treatments such as photocoagulation, intravitreal injection of triamcinolone, or vitrectomy. Patients were included in the study independently of the size of the leakage area, retinal thickness (as determined by optical coherence tomography [OCT]), visual acuity, age, metabolic control, type of diabetes mellitus, or previous treatments such as photocoagulation, vitrectomy, or intravitreal triamcinolone injection performed beyond a 6-month period previously.

Types of DME: all

Prior laser treatment: all participants

Age: 64.1

Intervention: Test intervention IVB

Control intervention: N/A

Repeat treatment: 16 patients had 2 injections of IVB

Outcomes: Primary outcome: BCVA and retinal thickness 
Measurement of primary outcome: VA by Snellen testing and ETDRS standard charts at a $4 \mathrm{~m}$ distance retinal thickness measurement by OCT

Secondary outcome: IOP and fundus changes

Measurement of secondary outcome: IOP measurement device, slit-lamp examination, and stereoscopic biomicroscopy of the retina using a 78-diopter lens

Data source: published data

Funding source: From the Department of Ophthalmology, Ludwig-Maximilians-University, Munich, Germany.

Country: Germany

Reason for exclusion: nonrandomized clinical trial.

\section{Chun et $\mathrm{al}^{32}$}

Title: A pilot study of multiple intravitreal injections of ranibizumab in patients with center-involving clinically significant diabetic macular edema

Methods: Study design: single-center, open-label, doseescalating pilot study

Method of randomization: N/A

Number of randomization: N/A

Number studied: 10 eyes

Method of allocation concealment: N/A

Outcome assessor masking: N/A

Losses to follow-up: Nil

Intention to treat analysis: Not performed

Participants: Inclusion criteria: patients over 18 years of age (male or female) with a history of diabetes mellitus who showed stereo fundus photographic evidence of centerinvolving clinically significant DME in the study eye within 28 days of the start of the study. Patients able to defer laser treatment for over 4 months.

Exclusion criteria: exclusively small $<100 \mu \mathrm{m}$ at the maximum dimension focal areas of edema in the study eye, central macular damage likely to preclude improvement in visual acuity (VA), previous use of intraocular steroids, previous participation in another study of antiangiogenic drugs, other ocular disorders that may confound interpretation of study results, ocular inflammation in the study eye, and history of vitreoretinal surgery in the study eye.

Type of DME: CSME

Age: 65.6 in $0.3 \mathrm{mg}$ IVR group and 73.0 in $0.5 \mathrm{mg}$ IVR group

Intervention: Test intervention: $0.3 \mathrm{mg}$ or $0.5 \mathrm{mg}$ of ranibizumab

Control intervention: N/A

Repeat treatment: 1 and 2 months
Outcomes: Primary outcome: BCVA and retinal thickness

Measurement of primary outcome: VA measured by ETDRS standard and retinal thickness measurement by OCT

Secondary outcome: macula leakage, drug tolerability.

Measurement of secondary outcome: fluorescein angiography (FA) to determine macular leakage, patients' measures and withdrawal measured tolerability.

Data source: published data

Funding source: Ophthalmic Consultants of Boston, Boston, Massachusetts. New England Eye Center, Tufts-New England Medical Center, Boston, Massachusetts. Genentech, Inc., South San Francisco, California. Dr Heier is a member of the Genentech Lucentis Advisory Board.

Country: United States of America

Unpublished ongoing studies

Reason for exclusion: nonrandomized clinical trial.

\section{Characteristics of ongoing studies Kriechbaum}

Title: A randomized, double-masked study with Intraocular bevacizumab (Avastin ${ }^{\circledR}$ ) compared with intraocular triamcinolone $\left(\right.$ Volon $\mathrm{A}^{\circledR}$ ) in patients with clinical significant diabetic macular edema

Method: randomized double blind trial

Intervention: $2.5 \mathrm{mg}$ IVB compared to $8 \mathrm{mg}$ IVT

Inclusion criteria: patients with diabetic macular edema with center involvement of CMT (macular edema) of at least 300 microns in the central subfield as measured by OCT; best-corrected visual acuity, using ETDRS charts, of 20/25 to 20/400 (Snellen equivalent) in the study eye; patients with decrease in vision in the study eye due to foveal thickening from diabetic macular edema and not to other causes, in the opinion of the investigator; patients without a necessity for panretinal laser photocoagulation for at least 3 months after study inclusion

Exclusion criteria: a condition that would preclude a patient for participation in the study in opinion of investigator, eg, unstable medical status including glycemic control and blood pressure. History of systemic corticosteroids within 3 months prior to randomization or topical, rectal, or inhaled corticosteroids in current use more than 3 times per week.

Prior/concomitant treatment: macular laser photocoagulation, panretinal laser photocoagulation within the past 3 months, previous treatment with intravitreal or sub-Tenon triamcinolone within the past 3 months in the 
study eye, previous participation in clinical trial involving antiangiogenic drugs (pegaptanib sodium, ranibizumab, anecortave acetate, protein kinase $\mathrm{C}$ inhibitor, etc), history of submacular surgery or other surgical intervention for diabetic macular edema in the study eye diabetic retinopathy characteristics, high risk proliferative diabetic retinopathy in the study eye without complete panretinal lasercoagulation and having a risk for intravitreal bleeding, concurrent ocular conditions, active intraocular inflammation (grade trace or above) in either eye, vitreomacular traction in the study eye evident by OCT, ocular disorders in the study eye including retinal vascular occlusion, retinal detachment, macular hole, choroidal neovascularization, intraocular surgery (including cataract surgery, YAG laser capsulotomy) in the study eye within 3 months preceding Day 0, uncontrolled glaucoma in the study eye (defined as IOP $\geq 25 \mathrm{mmHg}$ despite treatment with antiglaucoma medication), history of glaucoma filtration surgery, corneal transplantation in the study eye concurrent systemic conditions, history of myocardial infarction (in anamnesis or signs in ECG), history of congestive heart failure, history of stroke or transient ischemic attacks, significant abnormalities on laboratory testing (signs on failure of kidney, liver disease), premenopausal women not using adequate contraception and pregnant or nursing women and history of other disease, metabolic dysfunction, physical examination finding, or clinical laboratory finding giving reasonable suspicion of a disease or condition that contraindicates the use an investigational drug or that might affect interpretation of the results of the study or render the subject at high risk for treatment complications.

Primary outcome measure: BCVA and CMT

Secondary measures: structural mechanisms of DME by fluorescein angiography.

Data source: online clinical trials.gov register

Funding source: Medical University of Vienna Country: Austria

\section{Jorge}

Title: Intravitreal bevacizumab versus intravitreal triamcinolone acetonide for refractory diabetic macular edema

Methods: randomized controlled trial

Intervention: $2.5 \mathrm{mg}$ IVB compared to IVT $4 \mathrm{mg}$

Inclusion criteria: refractory diffuse DME (defined herein as clinically significant DME (by biomicroscopic evaluation) unresponsive to focal laser photocoagulation (performed at least 3 months before evaluation) and generalized breakdown of the inner blood-retina barrier with diffuse fluorescein leakage involving the foveal center and most of the macular area on fluorescein angiography), and Snellen logarithm of minimum angle of resolution (LogMAR) BCVA equivalent of 20/40 or worse, and CMT greater than $300 \mu \mathrm{m}$ on OCT.

Exclusion criteria: aphakic or pseudophakic eyes, glycosylated hemoglobin (HbA1C) rate above 10\%, history of glaucoma or ocular hypertension, loss of vision as a result of other causes, systemic corticoid therapy, history of thromboembolic event (including myocardial infarction or cerebral vascular accident), major surgery within the prior 6 months or planned within the next 28 days, uncontrolled hypertension (according to guidelines of the seventh report of the joint National Committee on prevention, detection, evaluation, and treatment of high blood pressure, 16 known coagulation abnormalities or current use of anticoagulative medication other than aspirin, severe systemic disease.

Primary outcome: BCVA and CMT by OCT

Secondary outcome: IOP

Data source: online clinical trials.gov register

Funding source: University of Sao Paulo

Country: Brazil

\section{Novartis}

Title: A randomized, double-masked, multicenter, Phase II study assessing the safety and efficacy of two concentrations of ranibizumab (intravitreal injections) compared with nontreatment control for the treatment of diabetic macular edema with center involvement

Method: treatment, randomized, double-bnlind, placebo control, parallel assignment, safety/efficacy study.

Inclusion criteria: diabetic macular edema with center involvement in at least one eye, Type 1 or type 2 diabetes mellitus diagnosed 2 years prior to screening, and laser photocoagulation in the study eye can be withheld for at least 3 months after randomization.

Exclusion criteria: patients with uncontrolled systemic or ocular diseases, have any history of any intraocular surgery in the study eye within the past 6 months preceding screening and conditions that require chronic concomitant therapy with systemic or topical ocular corticosteroids.

Primary outcome measure: no data

Secondary outcome measure: no data

Data source: online clinical trials.gov register

Funding source: Novartis

Country: Switzerland 


\section{Belfort}

Title: A randomized, parallel group, masked clinical study to evaluate the efficacy of triamcinolone and bevacizumab through intravitreal injection with individual or simultaneous drugs to treatment of diabetic macular edema

Method: randomized, double blind (subject, investigator), active control, parallel assignment, efficacy study

Intervention: three arm trial a) $1.25 \mathrm{mg}$ IVB b) $4 \mathrm{mg}$ IVT c) $1.25 \mathrm{mg}$ IVB and $4 \mathrm{mg}$ IVT

Inclusion criteria: 18 years of age at least, diagnosis of diabetes mellitus type 1 or type 2 . any one of the following will be considered to be sufficient evidence that diabetes is present: current regular use of insulin for the treatment of diabetes or current regular use of oral hypoglycemic agents for the treatment of diabetes or diabetes as defined by American Diabetes Association (ADA) or symptoms of diabetes (polyuria, polydipsia, and unexplained weight loss) or eigth-hour fasting plasma glucose $>126 \mathrm{mg} / \mathrm{dL}$. Diabetic macular edema clinically observable associated with diabetic retinopathy: without prior foveal treatment with laser therapy, if photocoagulation or peripherical or macular laser, at least 3 months or absence of macular ischemia by fluorescein angiography on baseline visit. BCVA score between 20 letters (20/400 ETDRS) e 70 letters (20/40 ETDRS) in the study eye measured by the ETDRS method at qualification/baseline visit. Retinal thickness $>275 \mu \mathrm{m}$ by OCT. One eye per patient will be chosen for the study. In case of both eye eligible, it will be chosen an eye to be treated with study medication and another eye treated with laser and taught hyaloid syndrome. Exclusion criteria: Uncontrolled systemic disease, initiation of medical therapy for diabetes or a change from oral hypoglycemic agents to insulin therapy within 4 months prior to the qualification visit. Renal failure requiring hemodialysis or peritoneal dialysis within 6 months prior to the qualification visit or any ocular condition in the study eye that in the opinion of the investigator would prevent a 2 lines improvement of visual acuity (eg, severe macular ischemia). Presence of branch retinal vein occlusion, central retinal vein occlusion, uveitis, pseudophakic cystoid edema or any other condition in the study eye which could be contributing to macular edema or presence of an epiretinal membrane in the study eye. History of IOP elevation in response to steroid treatment in either eye or glaucoma or optic nerve head change consistent with glaucoma damage. Ocular hypertension requiring more than one antiglaucoma medication to maintain IOP $<11 \mathrm{mmhg}$ at qualification visit, presence of anterior chamber intraocular lens in the study eye, active optic disc or retinal neovascularization in the study eye at qualification visit or active or history of choroidal neovascularization in the study eye.

Primary outcome: visual acuity

Secondary outcome: CRT by OCT and tonometry

Data source: clinical trials.gov register

Funding source: Federal University of Sao Paulo

Country: Brazil

\section{Adriana Solís-Vivanco}

Title: The efficacy of a single intravitreal injection of bevacizumab in patients with diffuse diabetic macular edema

Method: treatment, nonrandomized, open-label, uncontrolled, single group assignment, safety/efficacy study

Inclusion criteria: Type 2 diabetes, macular edema involving the center of the macula demonstrated on OCT, Clear ocular media, untreated patients, older than 45 years and BCVA of the fellow eye at least 20/100.

Exclusion criteria: Renal diabetic disease, uncontrolled hypertension or stroke history, other ocular disease, ocular surgery excepting uncomplicated phacoemulsification, history of photocoagulation (panretinal or focal), and a history of another intravitreal treatment (like triamcinolone).

Primary outcome measures: BCVA, fluorescein angiography, and optical coherence tomography

Data source: online clinical trials.gov register

Funding source: Asociacion para Evitar Ia Ceguera en Mexico

Country: Mexico

\section{Ute Wolf-Schnurrbusch}

Title: Short-term effects of intravitreal bevacizumab and triamcinolone in patients with diabetic macular edema

Method: observational

Study design: prospective case control

Participants: Inclusion criteria: patients with clinically significant macular edema

Exclusion criteria: retinal thickness $<250 \mu \mathrm{m}$

Primary outcome measure: central retinal thickness

Secondary outcome: BCVA

Data source: online clinical trials.gov register

Funding source: University Hospital Inselspital, Berne

Country: Switzerland

\section{Sheidow}

Title: Effect of Macugen ${ }^{\circledR}$ (pegaptanib) on surgical outcomes and growth factors including vascular endothelial growth factor (VEGF) levels in patients with proliferative diabetic 
retinopathy (PDR) and clinically significant diabetic macular edema (CSDME)

Method: nonrandomized, controlled trial

Intervention: $0.3 \mathrm{mg}$ of pegaptanib

Inclusion criteria: 18 years or older, type 1 or 2 diabetes, patients requiring surgical intervention for complications of proliferative diabetic retinopathy with vitreous hemorrhage or traction retinal detachment or clinically significant macular edema and women postmenopausal for 12 months before the study, surgically sterile, or not pregnant, and on effective contraception.

Exclusion criteria: previous retinal vein occlusion, any intraocular surgery within the previous 12 months, myopia of $>$ or $=$ to 8 diopters, and active ocular or periocular infection.

Primary outcome: levels of intravitreal Macugen postinjection of intravitreal Macugen.

Secondary outcome: Effect on ease of surgery post injection of intravitreal Macugen.

Data source: online clinical trials.gov register

Funding source: Pfizer $^{\circledR}$

Country: United Kingdom

\section{Lim}

Title: Avastin in the treatment of macular edema and uveitis

Method: nonrandomized trial

Intervention: $1.25 \mathrm{mg}$ IVB

Inclusion criteria: Clinically significant macular edema secondary to diabetes involving the fovea in one or both eyes that has been refractory to previous standard treatments (eg, laser) where local steroid therapy is contraindicated (eg, pre-existing glaucoma or steroid responder) or ineffective, OR 3. Uveitic cystoid macular edema in one or both eyes that has either been unresponsive to standard treatment (including intravitreal triamcinolone) or where further local steroid treatment is relatively contraindicated (eg, pre-existing glaucoma or steroid responder) or ineffective, OR 4. Subfoveal or juxtafoveal choroidal neovascularization (CNV) secondary to uveitis in one or both eyes, best-corrected visual acuity in the affected eye $(\mathrm{s})=$ $6 / 12$ or worse 5 . Subjects must have signed the informed consent form.

Exclusion criteria: Loss of vision due to other causes (eg, myopic macular degeneration), surgical intervention in the study eye within 2 months preceding recruitment, significant macular ischemia, no useful vision in fellow eye, known allergies to bevacizumab or ranibizumab, active ocular infection (eg, conjunctivitis, keratitis), intercurrent severe disease such as septicaemia, history of other systemic disease(s) that, in the opinion of the investigator, may render the subject at a high risk for treatment complications, any condition which would affect follow-up or photographic documentation (eg, geographical, psycho-social, media opacities), unwillingness or inability to give informed consent, under age 18, pregnant or lactating women, and premenopausal women.

Primary outcome: best-corrected, Early Treatment Diabetic Retinopathy Study (ETDRS), visual acuity

Secondary outcome: CMT as measured on OCT

Data source: Australian New Zealand Trials Registry

Funding source: Royal Victorian Eye and Ear Hospital Country: Australia
Clinical Ophthalmology

\section{Publish your work in this journal}

Clinical Ophthalmology is an international, peer-reviewed journal covering all subspecialties within ophthalmology. Key topics include: Optometry; Visual science; Pharmacology and drug therapy in eye diseases; Basic Sciences; Primary and Secondary eye care; Patient Safety and Quality of Care Improvements. This journal is indexed on

\section{Dovepress}

PubMed Central and CAS, and is the official journal of The Society of Clinical Ophthalmology (SCO). The manuscript management system is completely online and includes a very quick and fair peer-review system, which is all easy to use. Visit http://www.dovepress.com/ testimonials.php to read real quotes from published authors. 